\title{
Spectral Analysis of the Dirac Polaron
}

\author{
Dedicated to Professor Asao Arai on the occasion of his 60th birthday
}

\author{
by
}

Itaru SASAKI

\begin{abstract}
A system of a Dirac particle interacting with the radiation field is considered. The Hamiltonian of the system is defined by $H=\boldsymbol{\alpha} \cdot(\hat{\mathbf{p}}-q \mathbf{A}(\hat{\mathbf{x}}))+m \beta+H_{f}$, where $q \in \mathbb{R}$ is a coupling constant, $\mathbf{A}(\hat{\mathbf{x}})$ the quantized vector potential and $H_{f}$ the free photon Hamiltonian. Since the total momentum is conserved, $H$ is decomposed with respect to the total momentum with fiber Hamiltonian $H(\mathbf{p})\left(\mathbf{p} \in \mathbb{R}^{3}\right)$. Since the self-adjoint operator $H(\mathbf{p})$ is bounded from below, one can define the lowest energy $E(\mathbf{p}, m):=\inf \sigma(H(\mathbf{p}))$. We prove that $E(\mathbf{p}, m)$ is an eigenvalue of $H(\mathbf{p})$ under the following conditions: (i) infrared regularization and (ii) $E(\mathbf{p}, m)<E(\mathbf{p}, 0)$. We also discuss polarization vectors and the angular momentums.
\end{abstract}

2010 Mathematics Subject Classification: Primary 81Q10; Secondary 47B25.

Keywords: quantum electrodynamics, ground state, Dirac polaron, Dirac operator.

\section{$\S 1$. Introduction}

We consider a quantum system of a Dirac particle interacting with the radiation field. An example of a Dirac particle is the free electron. The Hilbert space for the Dirac particle is

$$
\mathcal{H}_{\mathrm{p}}:=L^{2}\left(\mathbb{R}_{\mathbf{x}}^{3} ; \mathbb{C}^{4}\right),
$$

and the free Hamiltonian for the Dirac particle is the free Dirac operator $\boldsymbol{\alpha} \cdot \hat{\mathbf{p}}+m \beta$ acting on $\mathcal{H}_{\mathrm{p}}$, where $\hat{\mathbf{p}}=-i \nabla_{\mathbf{x}}$ denotes the momentum for the Dirac particle. The Hilbert space for the radiation field is the Fock space:

$$
\mathcal{F}_{\text {rad }}:=\bigoplus_{n=0}^{\infty} \bigotimes_{\text {sym }}^{n} L^{2}\left(\mathbb{R}_{\mathbf{k}}^{3} \times\{1,2\}\right)
$$

Communicated by N. Ozawa. Received May 17, 2013. Revised October 21, 2013.

I. Sasaki: Department of Mathematical Sciences, Shinshu University, Matsumoto 390-8621, Japan;

e-mail: isasaki@shinshu-u.ac.jp

(C) 2014 Research Institute for Mathematical Sciences, Kyoto University. All rights reserved. 
where $\bigotimes_{\text {sym }}^{n}$ means the $n$-fold symmetric tensor product with $\bigotimes_{\text {sym }}^{0} L^{2}\left(\mathbb{R}_{\mathbf{k}}^{3} \times\right.$ $\{1,2\}):=\mathbb{C}$. The Hilbert space for the total system is defined by

$$
\mathcal{H}:=\mathcal{H}_{\mathrm{p}} \otimes \mathcal{F}_{\text {rad }} .
$$

In this paper, we consider the quantum system described by the Hamiltonian

$$
H:=\boldsymbol{\alpha} \cdot(\hat{\mathbf{p}}-q \mathbf{A}(\hat{\mathbf{x}}))+m \beta+H_{f},
$$

where $q \in \mathbb{R}$ is a coupling constant, $\mathbf{A}(\hat{\mathbf{x}})$ denotes the quantized magnetic vector potential in the Coulomb gauge and $H_{f}$ is the free photon Hamiltonian. We impose an ultraviolet cutoff in the quantized vector potential. We call the quantum system defined by (1.4) the Dirac-Maxwell model. The Hamiltonian (1.4) was introduced and discussed in the early days of quantum theory (see, e.g., [He]). By an informal perturbation theory, the Klein-Nishina formula (which gives a differential cross section for the Compton scattering) can be derived from the Dirac-Maxwell model [He]. Mathematical analysis of the Dirac-Maxwell model was initiated by A. Arai [A1, A2]. In [A3], A. Arai proved that a non-relativistic limit of the Dirac-Maxwell model converges to the Pauli-Fierz model (the non-relativistic QED). See also [A4]. The essential self-adjointness of the Hamiltonian (1.4) with an external potential was discussed by E. Stockmeyer and H. Zenk [SZ].

Since the Hamiltonian $H$ is translation invariant, the total momentum of the system is conserved, i.e., the Hamiltonian of the system strongly commutes with the total momentum operator

$$
\mathbf{P}:=\hat{\mathbf{p}}+d \Gamma(\mathbf{k}),
$$

where $d \Gamma(\mathbf{k})$ denotes the momentum operator of the radiation field. Hence the Hamiltonian can be decomposed as

$$
\begin{aligned}
& H \cong \int_{\mathbb{R}^{3}}^{\oplus} H(\mathbf{p}) d \mathbf{p}, \\
& \mathbf{P} \cong \int_{\mathbb{R}^{3}}^{\oplus} \mathbf{p} d \mathbf{p},
\end{aligned}
$$

where $\cong$ means unitary equivalence. In this paper, we mainly study the fiber Hamiltonian $H(\mathbf{p})$ which describes the dynamics of the relativistic particle dressed in photons with total momentum $\mathbf{p}$. We call the quantum system described by $H(\mathbf{p})$ the Dirac polaron. As shown in [A2, A1], for $\mathbf{p} \in \mathbb{R}^{3}, H(\mathbf{p})$ has the form

$$
H(\mathbf{p})=\boldsymbol{\alpha} \cdot \mathbf{p}+m \beta+H_{f}-\boldsymbol{\alpha} \cdot d \Gamma(\mathbf{k})-q \boldsymbol{\alpha} \cdot \mathbf{A},
$$

which acts on $\mathbb{C}^{4} \otimes \mathcal{F}_{\text {rad }}$, where $\mathbf{A}$ denotes the quantized vector potential at the origin $(=\mathbf{A}(\mathbf{0}))$. The fourth term $-\boldsymbol{\alpha} \cdot d \Gamma(\mathbf{k})$ describes the reaction due to the 
radiation field, and the last term $-q \boldsymbol{\alpha} \cdot \mathbf{A}$ is the electromagnetic interaction. It should be noted that $-q \boldsymbol{\alpha} \cdot \mathbf{A}$ is not $\left.H(\mathbf{p})\right|_{q=0}$-bounded for any non-zero $q$, because the reaction term $-\boldsymbol{\alpha} \cdot d \Gamma(\mathbf{k})$ is comparable to $H_{f}$, and $-q \boldsymbol{\alpha} \cdot \mathbf{A}$ is unbounded. This fact implies that $-q \boldsymbol{\alpha} \cdot \mathbf{A}$ is not a small perturbation no matter how small $q$ is. One of the important facts about the Dirac polaron is that $H(\mathbf{p})$ is bounded from below for all values of all constants: the total momentum $\mathbf{p}$, the mass $m$ and the coupling constant $q$ (see [S1]). Hence, one can define the lowest energy by

$$
E(\mathbf{p}, m):=\inf \sigma(H(\mathbf{p}))>-\infty,
$$

where $\sigma(A)$ denotes the spectrum of $A$. If $H(\mathbf{p})$ has an eigenvalue $E$ for $q \neq 0$, we say that a dressed particle state exists and the corresponding eigenvector is called a dressed particle state. In Section 4, we show that a dressed particle state exists under suitable conditions including (i) infrared regularization and (ii) the inequality

$$
E(\mathbf{p}, m)<E(\mathbf{p}, 0)
$$

The condition (1.10) will be assumed in Theorems 4.1, 4.2 and 4.4 below. One can observe that there exist $m^{*}>0$ such that (1.10) holds for all $|m|>m^{*}$. We expect that $m^{*}=0$, but we have no proof. In Section 5 , we study the angular momentum and degeneracy of eigenvalues of the Dirac polaron $H(\mathbf{p})$. We will show that the angular momentum of the p-direction commutes with $H(\mathbf{p})$, and any eigenvalue of $H(\mathbf{p})$ has an even multiplicity (admitting infinity). Therefore $E(\mathbf{p}, m)$ is degenerate if it is an eigenvalue of $H(\mathbf{p})$.

This paper has three appendices. In Appendix A, we show that all spectral properties of the Dirac-Maxwell model and the Dirac polarons are independent of the choice of polarization vectors. Namely, two Hamiltonians defined by different polarization vectors are unitarily equivalent. The discussion in Appendix A is applicable to various QED models (e.g., Pauli-Fierz model). In Appendix B, we propose a general definition of angular momentum. Although the spectral properties of QED Hamiltonians are independent of the choice of polarization vectors, the definition of angular momentum depends on these vectors.

In Appendix C, we show some properties of the lowest energy $E(\mathbf{p})$ which are used in the proofs of Theorems 4.1-4.4.

\section{§2. Definition of the model}

In this paper, unless confusion may arise, we omit the symbol " $\otimes$ " between two operators, for example, we write $A \otimes I$ as $A$ and $I \otimes B$ as $B$, where $I$ denotes the identity operator. For a closable operator $T$ on $L^{2}\left(\mathbb{R}_{\mathbf{k}}^{3} \times\{1,2\}\right)$, we denote 
by $d \Gamma(T)$ and $\Gamma(T)$ the second quantization operators of $T$ (see [RS2]), which act on $\mathcal{F}_{\text {rad. }}$. For $f \in L^{2}\left(\mathbb{R}_{\mathbf{k}}^{3} \times\{1,2\}\right)$, we denote by $a(f)$ and $a(f)^{*}$ the annihilation operator and the the creation operator, respectively (see [RS2]), which are closed operators acting on $\mathcal{F}_{\text {rad }}$. Let $\mathbf{e}^{(\lambda)}: \mathbb{R}^{3} \rightarrow \mathbb{R}^{3}, \lambda=1,2$, be polarization vectors:

$$
\mathbf{e}^{(\lambda)}(\mathbf{k}) \cdot \mathbf{e}^{(\mu)}(\mathbf{k})=\delta_{\lambda, \mu}, \quad \mathbf{e}^{(\lambda)}(\mathbf{k}) \cdot \mathbf{k}=\mathbf{0}, \quad \mathbf{k} \in \mathbb{R}^{3}, \lambda, \mu \in\{1,2\} .
$$

We write $\mathbf{e}^{(\lambda)}(\mathbf{k})=\left(e_{1}^{(\lambda)}(\mathbf{k}), e_{2}^{(\lambda)}(\mathbf{k}), e_{3}^{(\lambda)}(\mathbf{k})\right)$, and we suppose that each component $e_{j}^{(\lambda)}(\mathbf{k})$ is a Borel measurable function of $\mathbf{k}$. For objects $\mathbf{a}=\left(a_{1}, a_{2}, a_{3}\right)$ and $\mathbf{b}=\left(b_{1}, b_{2}, b_{3}\right)$, we set $\mathbf{a} \cdot \mathbf{b}:=\sum_{j=1}^{3} a_{j} b_{j}$. For a linear $F(\cdot)$ we set $F(\mathbf{a}):=$ $\left(F\left(a_{1}\right), F\left(a_{2}\right), F\left(a_{3}\right)\right)$. Let $\omega$ be multiplication by the function

$$
\omega(\mathbf{k})=|\mathbf{k}| .
$$

We choose a function

$$
\hat{\rho} \in L^{2}\left(\mathbb{R}_{\mathbf{k}}^{3}\right) \cap \operatorname{Dom}\left(\omega^{-1}\right),
$$

where Dom means operator domain. For $j=1,2,3$ and $\mathbf{x} \in \mathbb{R}^{3}$, we set

$$
g_{j}(\mathbf{k}, \lambda ; \mathbf{x}):=|\mathbf{k}|^{-1 / 2} \hat{\rho}(\mathbf{k}) e_{j}^{(\lambda)}(\mathbf{k}) e^{-i \mathbf{k} \cdot \mathbf{x}}, \quad(\mathbf{k}, \lambda) \in \mathbb{R}_{\mathbf{k}}^{3} \times\{1,2\} .
$$

For each fixed $\mathbf{x} \in \mathbb{R}^{3}$, the function $g_{j}(\mathbf{x})(\cdot):=g_{j}(\cdot ; \mathbf{x})$ is in $L^{2}\left(\mathbb{R}_{\mathbf{k}}^{3} \times\{1,2\}\right)$. The quantized magnetic vector potential at $\mathbf{x} \in \mathbb{R}^{3}$ is defined by

$$
\begin{aligned}
\mathbf{A}(\mathbf{x}) & \left.:=\left(A_{1}(\mathbf{x})\right), A_{2}(\mathbf{x}), A_{3}(\mathbf{x})\right), \\
A_{j}(\mathbf{x}) & :=\frac{1}{\sqrt{2}} \overline{\left[a\left(g_{j}(\mathbf{x})\right)+a\left(g_{j}(\mathbf{x})\right)^{*}\right]}, \quad j=1,2,3,
\end{aligned}
$$

where, for a closable operator $T, \bar{T}$ denotes its closure. For each $\mathbf{x} \in \mathbb{R}^{3}, A_{j}(\mathbf{x})$ is a self-adjoint operator on $\mathcal{F}_{\text {rad }}$ (see $[\mathrm{RS} 2]$ ). Since $\mathbf{e}^{(\lambda)}(\mathbf{k})$ 's are perpendicular to $\mathbf{k}$, the operators $\mathbf{A}(\mathbf{x})$ satisfy the Coulomb gauge condition

$$
\operatorname{div} \mathbf{A}(\mathbf{x})=\sum_{j=1}^{3} \partial_{x_{j}} A_{j}(\mathbf{x})=0
$$

Remark 2.1. The function $\hat{\rho}$ is called an ultraviolet cutoff function. A typical example of $\hat{\rho}$ is the characteristic function of the region $\left\{\mathbf{k} \in \mathbb{R}^{3}|\kappa \leq| \mathbf{k} \mid \leq \Lambda\right\}$, where $\kappa$ and $\Lambda$ are non-negative constants. Here $\Lambda$ is called an ultraviolet cutoff, and $\kappa$ is an infrared cutoff if it is strictly positive.

The Hilbert space $\mathcal{H}$ can be identified as

$$
\mathcal{H}=L^{2}\left(\mathbb{R}_{\mathbf{x}}^{3} ; \mathbb{C}^{4} \otimes \mathcal{F}_{\text {rad }}\right)=\int_{\mathbb{R}^{3}}^{\oplus} \mathbb{C}^{4} \otimes \mathcal{F}_{\text {rad }} d \mathbf{x} .
$$


Under this identification, we define the quantized vector potential in the following way. Since $g_{j}(\mathbf{x}) \in L^{2}\left(\mathbb{R}_{\mathbf{k}}^{3} \times\{1,2\}\right)$ is strongly continuous in $\mathbf{x} \in \mathbb{R}^{3}$, the map $\mathbf{x} \mapsto A_{j}(\mathbf{x})$ is a self-adjoint operator valued measurable function. Then we can define a self-adjoint operator on $\mathcal{H}$ by

$$
A_{j}(\hat{\mathbf{x}}):=\int_{\mathbb{R}^{3}}^{\oplus} A_{j}(\mathbf{x}) d \mathbf{x}
$$

Namely, when we identify $\Psi \in D\left(A_{j}(\hat{\mathbf{x}})\right)$ with an $\mathcal{F}_{\text {rad-valued square integrable }}$ function, the action of the operator $A_{j}(\hat{\mathbf{x}})$ is given by $\left(A_{j}(\hat{\mathbf{x}}) \Psi\right)(\mathbf{x})=A_{j}(\mathbf{x}) \Psi(\mathbf{x})$, $\mathbf{x} \in \mathbb{R}^{3}$. The operator valued vector

$$
\mathbf{A}(\hat{\mathbf{x}}):=\left(A_{1}(\hat{\mathbf{x}}), A_{2}(\hat{\mathbf{x}}), A_{3}(\hat{\mathbf{x}})\right)
$$

is also called the quantized vector potential.

The free photon Hamiltonian is the second quantization of $\omega$ :

$$
H_{f}:=d \Gamma(\omega) .
$$

The Dirac-Maxwell Hamiltonian is defined by

$$
H:=\boldsymbol{\alpha} \cdot(\hat{\mathbf{p}}-q \mathbf{A}(\hat{\mathbf{x}}))+m \beta+H_{f},
$$

where $\hat{\mathbf{p}}=-i \nabla_{\mathbf{x}}$ and $\nabla_{\mathbf{x}}$ is the gradient operator acting in $\mathcal{H}_{\mathrm{p}}, \boldsymbol{\alpha}=\left(\alpha_{1}, \alpha_{2}, \alpha_{3}\right)$ and $\beta$ are Dirac matrices satisfying $\alpha_{1}, \alpha_{2}, \alpha_{3}, \beta \in M_{4}(\mathbb{C})$ and

$$
\begin{aligned}
& \alpha_{j} \alpha_{k}+\alpha_{k} \alpha_{j}=2 \delta_{j k}, \\
& \alpha_{j} \beta+\beta \alpha_{j}=0, \\
& \beta^{2}=I_{\mathbb{C}^{4}},
\end{aligned}
$$

the constant $m \in \mathbb{R}$ is the rest mass of the Dirac particle, and $q \in \mathbb{R}$ is a coupling constant. On the right hand side of (2.8), we omit the symbols $\otimes I$ and $I \otimes$, i.e., (2.8) is an abbreviation for

$$
H=(\boldsymbol{\alpha} \cdot \hat{\mathbf{p}}+m \beta) \otimes I_{\mathcal{F}_{\text {rad }}}-q \sum_{j=1}^{3}\left(\alpha_{j} \otimes I_{L^{2}\left(\mathbb{R}_{\mathbf{x}}^{3}\right)}\right) \cdot A_{j}(\hat{\mathbf{x}})+I_{\mathcal{H}_{\mathrm{p}}} \otimes H_{f} .
$$

In this paper, we use the Weyl representation for the Dirac matrices. Since all representations of the Dirac matrices are unitarily equivalent to each other, this choice does not affect the spectral properties of $H$ (see [T, Lemma 2.25]).

It is easy to see that $H$ is symmetric. Although the essential self-adjointness of $H$ was proven in [A1], we give a slightly improved result:

Proposition 2.2 (Essential self-adjointness). $\bar{H}$ is a self-adjoint operator and essentially self-adjoint on any core for $\sqrt{-\triangle}+H_{f}$. 
Proof. The proof is a simple application of Nelson's commutator theorem. Our choice of a comparison operator for Nelson's commutator theorem is $\sqrt{-\triangle}+H_{f}$. See [S2] for details.

\section{§3. Momentum conservation and fiber Hamiltonian $H(\mathbf{p})$}

The total momentum operator is defined by

$$
\mathbf{P}:=\overline{\hat{\mathbf{p}}+d \Gamma(\mathbf{k})} \text {. }
$$

The Hamiltonian $H$ strongly commutes with $\mathbf{P}$ (see [A1]). To construct the fiber Hamiltonian, we define a self-adjoint operator

$$
Q:=\overline{\mathbf{x} \cdot d \Gamma(\mathbf{k})}
$$

Let $U_{F}$ be the Fourier transform from $L^{2}\left(\mathbb{R}_{\mathbf{x}}^{3}\right)$ to $L^{2}\left(\mathbb{R}_{\mathbf{p}}^{3}\right)$. We set

$$
U:=\left(U_{F} \otimes I_{\mathbb{C}^{4}}\right) \exp (i Q) .
$$

Then we can identify $U \mathcal{H}$ as a constant fiber direct integral

$$
U \mathcal{H} \cong \int_{\mathbb{R}^{3}}^{\oplus} \mathbb{C}^{4} \otimes \mathcal{F}_{\text {rad }} d \mathbf{p}
$$

For every $\mathbf{p} \in \mathbb{R}^{3}$, we define

$$
H(\mathbf{p}):=\boldsymbol{\alpha} \cdot \mathbf{p}+m \beta+H_{f}-\boldsymbol{\alpha} \cdot d \Gamma(\mathbf{k})-q \boldsymbol{\alpha} \cdot \mathbf{A},
$$

which acts on $\mathbb{C}^{4} \otimes \mathcal{F}_{\text {rad }}$, where $\mathbf{A}:=\mathbf{A}(\mathbf{0})$.

Proposition 3.1. For all $\mathbf{p} \in \mathbb{R}^{3}, H(\mathbf{p})$ is essentially self-adjoint and

$$
\begin{aligned}
U \bar{H} U^{*} & =\int_{\mathbb{R}^{3}}^{\oplus} \overline{H(\mathbf{p})} d \mathbf{p}, \\
U \mathbf{P} U^{*} & =\int_{\mathbb{R}^{3}}^{\oplus} \mathbf{p} d \mathbf{p},
\end{aligned}
$$

where $\int^{\oplus}(\cdots)$ denotes the fiber direct integral operator with respect to (3.4).

Proof. See [A2].

Remark 3.2. Physically $\overline{H(\mathbf{p})}$ is the Hamiltonian of the fixed total momentum $\mathbf{p} \in \mathbb{R}^{3}$. One can show that the spectral properties of $\overline{H(\mathbf{p})}$ are independent of the choice of polarization vectors, because the Hamiltonians with different polarization vectors are unitarily equivalent. See Appendix A. 
Remark 3.3. We call $H(\mathbf{p})$ the Dirac polaron Hamiltonian; it was introduced in [A4]. It is expected that, as in the model of the H. Fröhlich polaron, electromagnetic interaction forms a quasiparticle where the bare Dirac particle is surrounded by photon clouds. Such a quasiparticle with momentum $\mathbf{p} \in \mathbb{R}^{3}$ is considered as a ground state of $\overline{H(\mathbf{p})}$, if it exists. The existence of a ground state of $\overline{H(\mathbf{p})}$ is the main subject of our paper.

Remark 3.4. Note that $\operatorname{Dom}(\boldsymbol{\alpha} \cdot d \Gamma(\mathbf{k})) \subset \operatorname{Dom}\left(H_{f}\right)$. Hence we have $\operatorname{Dom}\left(H_{f}\right)=$ $\operatorname{Dom}(H(\mathbf{p}))$ and $H(\mathbf{p})$ is essentially self-adjoint on $\operatorname{Dom}\left(H_{f}\right)$.

One of the most important properties of $\overline{H(\mathbf{p})}$ is semi-boundedness:

Theorem 3.5 ([S1]). For any $\mathbf{p}, \overline{H(\mathbf{p})}$ is bounded from below. Moreover $H(\mathbf{p})$ is essentially self-adjoint on any core for $H_{f}$.

Proof. The first statement was shown in [S1], where it is assumed that $\hat{\rho} \in$ $\operatorname{Dom}\left(\omega^{1 / 2}\right)$, but one can remove this condition by the following procedure. In [S1, ineq. (24)], it is shown that $H(\mathbf{p})$ is bounded from below, and the lower bound is a function of $\left\|\omega^{1 / 2} \mathbf{g}\right\|_{L^{2}\left(\mathbb{R}^{3}\right)}$ and not $\|\omega \mathbf{g}\|_{L^{2}\left(\mathbb{R}^{3}\right)}$. Therefore, firstly, we regularize $\hat{\rho}$ as $\hat{\rho}_{\lambda}(\mathbf{k}):=\hat{\rho}(\mathbf{k}) \chi_{|\mathbf{k}| \leq \lambda}$, and then we obtain the lower bound of the regularized Hamiltonian $H_{\lambda}(\mathbf{p}) \geq C_{\epsilon}$. Since $C_{\lambda}$ converges as $\lambda \rightarrow \infty$ and $H_{\mathbf{p}}$ converges to $H(\mathbf{p})$ on a finite particle subspace, we get $H(\mathbf{p}) \geq \lim _{\epsilon \rightarrow+0} C_{\epsilon}>-\infty$. The second statement follows from Wüst's Theorem [RS2] and the bound

$$
\|\boldsymbol{\alpha} \cdot(d \Gamma(\mathbf{k})-q \mathbf{A}) \Psi\|^{2} \leq\left\|\left(H_{f}+E\right) \Psi\right\|^{2}, \quad \Psi \in \operatorname{Dom}\left(H_{f}\right),
$$

for some $E>0$. The bound (3.8) was given in [S1].

Thus we can define the lowest energy of the Dirac polaron with total momentum $\mathbf{p}$ by

$$
E(\mathbf{p}, m):=\inf \sigma(\overline{H(\mathbf{p})})
$$

The energy $E(\mathbf{p}, m)$ depends on all parameters $(\mathbf{p}, m, q) \in \mathbb{R}^{3} \times \mathbb{R} \times \mathbb{R}$. When the $m$-dependence in $E(\mathbf{p}, m)$ is not important, we write $E(\mathbf{p}, m)$ as $E(\mathbf{p})$.

\section{§4. Existence of a ground state}

For a self-adjoint operator bounded below, $T$, we say that $T$ has a ground state if $\inf \sigma(T)$ is an eigenvalue of $T$. In this section, we give criteria for $\overline{H(\mathbf{p})}$ to have a ground state. 
Theorem 4.1. Suppose that $\hat{\rho}$ is spherically symmetric and

$$
\int_{\mathbb{R}^{3}} \frac{q^{2}}{(E(\mathbf{p}-\mathbf{k})-E(\mathbf{p})+|\mathbf{k}|)^{2}} \frac{|\hat{\rho}(\mathbf{k})|^{2}}{|\mathbf{k}|} d \mathbf{k}<1 .
$$

Assume that $E(\mathbf{p}, m)<E(\mathbf{p}, 0)$. Then the Dirac polaron Hamiltonian $\overline{H(\mathbf{p})}$ has a ground state.

Using the lower bound on $E(\mathbf{p}-\mathbf{k})-E(\mathbf{p})+|\mathbf{k}|$ which is proved in Theorem C.10 of Appendix C, we obtain the following result:

Theorem 4.2. Assume that $\hat{\rho}$ be spherically symmetric and $E(\mathbf{p}, m)<E(\mathbf{p}, 0)$. Assume the infrared regularity condition $\hat{\rho} \in \operatorname{Dom}\left(\omega^{-3 / 2}\right)$ holds. Then there exists a constant $q_{0}>0$ such that for all $q$ with $|q|<q_{0}, \overline{H(\mathbf{p})}$ has a ground state.

Remark 4.3. Since $E(\mathbf{p}, m)$ is concave in $m$ (Proposition C.1) and since we have $\lim _{m \rightarrow \infty} E(\mathbf{p}, m)=-\infty$, there exists $m^{*} \geq 0$ such that $E(\mathbf{p}, m)<E(\mathbf{p}, 0)$ for all $|m|>m^{*}$.

The proof of Theorem 4.1 is based on estimates of a photon number bound. The condition (4.1) can be considered as a restriction on the coupling constant $q$. There are two ways to remove this restriction. The first one is the method discovered by C. Gérard [Ge], and the other is the photon derivative bound developed in [GLL]. In this paper, we use the photon derivative bound. We need some additional assumptions:

( $\Lambda$ ) (i) $\hat{\rho}$ is a spherically symmetric function. (ii) There is an open set $S \subset \mathbb{R}^{3}$ such that $\bar{S}=\operatorname{supp} \hat{\rho}$ and $\hat{\rho}$ is continuously differentiable on $S$. (iii) For all $R>0$, the bounded region $S_{R}:=\{\mathbf{k} \in S|| \mathbf{k} \mid<R\}$ has the cone property (see [LL] for the definition).

The theorem below proves the existence of a ground state of the Dirac polaron for all values of the coupling constant $q$ :

Theorem 4.4. Assume that condition $(\Lambda)$ holds. Moreover assume that

$$
\hat{\rho} \in \operatorname{Dom}\left(\omega^{-3 / 2}\right), \quad|\mathbf{k}|^{-5 / 2} \hat{\rho}(\mathbf{k}) \in L^{p}\left(S_{R}\right), \quad|\mathbf{k}|^{-3 / 2}|\nabla \hat{\rho}(\mathbf{k})| \in L^{p}\left(S_{R}\right),
$$

for all $p \in[1,2)$ and $R>0$. Suppose that $E(\mathbf{p}, m)<E(\mathbf{p}, 0)$. Then $\overline{H(\mathbf{p})}$ has a ground state.

Remark 4.5. We now give an example. Let $\chi_{\kappa, \Lambda}(\mathbf{k})$ be the characteristic function of the region $\left\{\mathbf{k} \in \mathbb{R}^{3}|\kappa<| \mathbf{k} \mid<\Lambda\right\}$. For all $\kappa>0$ and $\Lambda<\infty$, the cutoff function $\hat{\rho}=\chi_{\kappa, \Lambda}$ satisfies $(\Lambda)$ and (4.2). The function $\hat{\rho}(\mathbf{k})=|\mathbf{k}| \exp (-\lambda|\mathbf{k}|)(\lambda>0)$ also satisfies condition $(\Lambda)$ and $(4.2)$. 
Remark 4.6. It is known that, in non-relativistic QED, the existence of a dressed particle requires the restriction $|\mathbf{p}| / m \leq 1$ (see [C]). On the other hand, Theorems 4.1-4.4 do not require a restriction on $|\mathbf{p}| / m$. This fact is a crucial difference between relativistic and non-relativistic dynamics. This result can be interpreted as follows. In general, the velocity operator is defined by $i=\sqrt{-1}$ times the commutator of the energy Hamiltonian with the position. Hence, the velocity operators of the non-relativistic particle and Dirac particle are defined by

$$
\begin{aligned}
\hat{\mathbf{p}} / m & =i\left[\hat{\mathbf{p}}^{2} / 2 m, \mathbf{x}\right], \\
\boldsymbol{\alpha} & =[\boldsymbol{\alpha} \cdot \hat{\mathbf{p}}+m \beta, \mathbf{x}],
\end{aligned}
$$

respectively. Hence the non-relativistic particle can move faster than light, and the particle with velocity $|\mathbf{p}| / m>1$ makes a shock wave of light and loses its kinetic energy. Therefore such a non-relativistic particle is unstable in the presence of electromagnetic interaction. On the other hand, since the speed of the Dirac particle is smaller than that of light, $\|\boldsymbol{\alpha}\| \leq 1$, this kind of catastrophe does not occur, and the dressed electron state is stable for all $|\mathbf{p}|$.

Remark 4.7. It is easy to see that the Hermitian matrix $\boldsymbol{\alpha} \cdot \mathbf{p}+m \beta$ has two eigenvalues $\pm \sqrt{\mathbf{p}^{2}+m^{2}}$, each of which is two-fold degenerate. Let $u_{i}^{( \pm)} \in \mathbb{C}^{4}$, $i=1,2$, be the corresponding normalized eigenvectors:

$$
(\boldsymbol{\alpha} \cdot \mathbf{p}+m \beta) u_{i}^{( \pm)}= \pm \sqrt{\mathbf{p}^{2}+m^{2}} u_{i}^{( \pm)}, \quad i=1,2 .
$$

Let $\Omega:=(1,0,0, \ldots) \in \mathcal{F}_{\text {rad }}$ be the vacuum. It is the unique eigenvector of both $H_{f}$ and $d \Gamma\left(k_{j}\right), j=1,2,3$. We set $\Phi_{i}^{( \pm)}:=u_{i}^{( \pm)} \otimes \Omega, j=1,2$. Clearly,

$$
\left.H(\mathbf{p})\right|_{q=0} \Phi_{i}^{( \pm)}= \pm \sqrt{\mathbf{p}^{2}+m^{2}} \Phi_{i}^{( \pm)}, \quad i=1,2 .
$$

Thus, in the case $q=0,\left.H(\mathbf{p})\right|_{q=0}$ has two eigenvalues $\pm \sqrt{\mathbf{p}^{2}+m^{2}}$. These eigenvectors $\Phi_{i}^{(+)}, i=1,2$ (resp. $\left.\Phi_{i}^{(-)}, i=1,2\right)$ describe states of a freely moving positive (resp. negative) energy particle with momentum p. Hence, if photons and the Dirac particle are decoupled, a Dirac particle associated with a positive eigenvalue exists and the positive eigenvalue is embedded. We are interested in the fate of those eigenvalues when interaction is switched on. As is shown in Fig. 1, the lowest energy $E(\mathbf{p}, m)$ converges to $-\sqrt{\mathbf{p}^{2}+m^{2}}$ as $q \rightarrow 0$. According to textbooks of physics (e.g. [B, He]), it is expected that any positive energy electron falls down to a negative energy state by a spontaneous emission of photons. Hence it is expected that the eigenvalue $+\sqrt{\mathbf{p}^{2}+m^{2}}$ is unstable under the perturbation $q \boldsymbol{\alpha} \cdot \mathbf{A}$. Theorems 4.1-4.4 ensure that a negative energy dressed electron exists under some conditions. But the instability of $\sqrt{\mathbf{p}^{2}+m^{2}}$ has not been proved yet. 


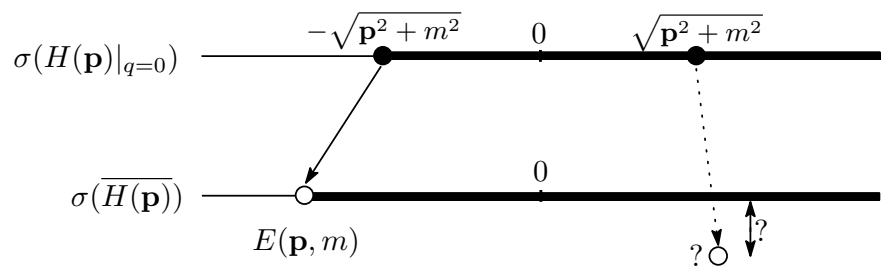

Figure 1. Spectrum of $\left.H(\mathbf{p})\right|_{q=0}$ and $H(\mathbf{p})$.

\section{$\S 5$. Angular momentum and degeneracy of eigenvalues}

In this section we show that the angular momentum around the $\mathbf{j}$-axis (where $\mathbf{j} \in \mathbb{R}^{3} \backslash\{0\}$ ) of the Dirac polaron is conserved if $\mathbf{p}$ is parallel to $\mathbf{j}$ and $\hat{\rho}(\mathbf{k})$ has axial symmetry around $\mathbf{j}$. Let $(\overline{H(\mathbf{p})}, \mathbf{e})$ be a Dirac polaron model with an arbitrarily given polarization vectors $\mathbf{e}=\left(\mathbf{e}^{(1)}, \mathbf{e}^{(2)}\right)$. The total angular momentum around the $\mathbf{j}$-axis in the system $(\overline{H(\mathbf{p})}, \mathbf{e})$ is defined by

$$
J_{\mathbf{j}}(\mathbf{e}):=S_{\mathbf{j}}+L_{\mathbf{j}}(\mathbf{e}),
$$

where $S_{\mathbf{j}}:=\bigoplus^{2}(\mathbf{j} \cdot \vec{\sigma}) / 2, \vec{\sigma}=\left(\sigma_{1}, \sigma_{2}, \sigma_{3}\right)$ are the Pauli matrices, and $L_{\mathbf{j}}(\mathbf{e})$ is an angular momentum for the radiation field, which is defined in Appendix B.

Proposition 5.1. The spectrum of $J_{\mathbf{j}}(\mathbf{e})$ is the set of half-integers:

$$
\sigma\left(J_{\mathbf{j}}(\mathbf{e})\right)=\mathbb{Z}_{1 / 2}:=\{ \pm 1 / 2, \pm 3 / 2, \pm 5 / 2, \ldots\} .
$$

In particular, $J_{\mathbf{j}}(\mathbf{e})$ decomposes as

$$
J_{\mathbf{j}}(\mathbf{e}) \cong \bigoplus_{z \in \mathbb{Z}_{1 / 2}} z
$$

with respect to the identification

$$
\mathbb{C}^{4} \otimes \mathcal{F}_{\text {rad }} \cong \bigoplus_{z \in \mathbb{Z}_{1 / 2}} \mathcal{F}(z)
$$

We conclude this section with the following:

Theorem 5.2. Let $\mathbf{j}$ be a unit vector parallel to $\mathbf{p}$. Assume that $\hat{\rho}(\mathbf{k})=\hat{\rho}(R \mathbf{k})$, $\mathbf{k} \in \mathbb{R}^{3}$, for all $R \in O(3)$ with $R \mathbf{j}=\mathbf{j}$. Then $\overline{H(\mathbf{p})}$ strongly commutes with $J_{\mathbf{j}}(\mathbf{e})$. In particular, $\overline{H(\mathbf{p})}$ decomposes as

$$
\overline{H(\mathbf{p})} \cong \bigoplus_{z \in \mathbb{Z}_{1 / 2}} H(\mathbf{p}: z),
$$

corresponding to the decomposition (5.1). Moreover, for all $z \in \mathbb{Z}_{1 / 2}, H(\mathbf{p}: z)$ is unitarily equivalent to $H(\mathbf{p}:-z)$, and the multiplicity of any eigenvalue of $\overline{H(\mathbf{p})}$ is even. 
Remark 5.3. In [Hi], F. Hiroshima defines an angular momentum in QED which differs from our definition.

\section{$\S 6$. Proof of Theorems 4.1-4.4}

For a constant $\nu \geq 0$, we define a regularized Hamiltonian to avoid the risk of infrared divergence:

$$
H_{\nu}(\mathbf{p}):=\boldsymbol{\alpha} \cdot \mathbf{p}+m \beta+H_{f}(\nu)-\boldsymbol{\alpha} \cdot d \Gamma(\mathbf{k})-q \boldsymbol{\alpha} \cdot \mathbf{A},
$$

where

$$
H_{f}(\nu):=d \Gamma\left(\omega_{\nu}\right), \quad \omega_{\nu}(\mathbf{k})=(1+\nu)|\mathbf{k}|+\nu .
$$

Let $N_{f}:=d \Gamma(1)$ be the photon number operator. Note that we have $H_{f}(\nu)=$ $H_{f}+\nu\left(H_{f}+N_{f}\right)$ and $H_{0}(\mathbf{p})=H(\mathbf{p})$. By the Kato-Rellich theorem, one can easily show that, for all $\nu>0, H_{\nu}(\mathbf{p})$ is self-adjoint on $\operatorname{Dom}\left(H_{f}(\nu)\right)$, and essentially selfadjoint on any core for $H_{f}(\nu)$. Since $H_{\nu}(\mathbf{p}) \geq H(\mathbf{p}) H_{\nu}(\mathbf{p})$ is also bounded from below. We set $\mathcal{D}:=\operatorname{Dom}\left(H_{f}\right) \cap \operatorname{Dom}\left(N_{f}\right)$. Then $\mathcal{D}$ is a common core for $\overline{H_{\nu}(\mathbf{p})}$ $(\nu \geq 0)$. We set

$$
E_{\nu}(\mathbf{p}):=\inf \sigma\left(\overline{H_{\nu}(\mathbf{p})}\right)
$$

For $\nu>0$, the massive Hamiltonian $H_{\nu}(\mathbf{p})$ was studied in [A1, A2], where A. Arai showed that $H_{\nu}(\mathbf{p})$ has a ground state for all $\nu>0$.

Lemma 6.1 (Existence of a ground state for $\nu>0$ ). Assume that $\nu>0$. Then

$$
\inf \sigma_{\mathrm{ess}}\left(H_{\nu}(\mathbf{p})\right)-E_{\nu}(\mathbf{p}) \geq \nu
$$

In particular, $H_{\nu}(\mathbf{p})$ has a ground state.

Proof. See [A2].

By Lemma 6.1, for all $\nu>0, H_{\nu}(\mathbf{p})$ has a normalized ground state $\Phi_{\nu}(\mathbf{p}) \in$ $\operatorname{Dom}\left(H_{f}(\nu)\right)$. In the following, we construct a ground state of $H_{0}(\mathbf{p})$ as a suitable limit of $\Phi_{\nu}(\mathbf{p})$. Since $\Phi_{\nu}(\mathbf{p})$ is normalized, there exists a sequence $\left\{\Phi_{\nu_{j}}(\mathbf{p})\right\}_{j=1}^{\infty}$ with $\lim _{j \rightarrow \infty} \nu_{j}=0$ such that $\left\{\Phi_{\nu_{j}}\right\}_{j}$ has a weak limit.

Lemma 6.2. Let $\left\{\nu_{j}\right\}_{j=1}^{\infty}$ be a sequence such that $\Phi_{\nu_{j}}$ has a weak limit $\Phi_{0}(\mathbf{p}):=$ $\mathrm{w}-\lim _{j \rightarrow \infty} \Phi_{\nu_{j}}$. Assume $\Phi_{0} \neq 0$. Then $\Phi_{0} \in \operatorname{Dom}(\overline{H(\mathbf{p})})$ and $\Phi_{0}$ is a ground state of $\overline{H(\mathbf{p})}$.

Proof. For all $\Psi \in \mathcal{D}$, one has

$$
\left\langle H(\mathbf{p}) \Psi, \Phi_{0}\right\rangle=\lim _{j \rightarrow \infty}\left\langle\Psi, H(\mathbf{p}) \Phi_{\nu_{j}}\right\rangle=\lim _{j \rightarrow \infty}\left\langle\Psi,\left\{E_{\nu_{j}}(\mathbf{p})-\nu_{j}\left(H_{f}+N_{f}\right)\right\} \Phi_{\nu_{j}}\right\rangle .
$$


By Proposition C.9, we have $E_{\nu_{j}}(\mathbf{p}) \rightarrow E_{0}(\mathbf{p})$ as $j \rightarrow \infty$. By assumption (2), we have

$$
\lim _{j \rightarrow \infty} \nu_{j}\left|\left\langle\Psi,\left(H_{f}+N_{f}\right) \Phi_{\nu_{j}}\right\rangle\right| \leq \lim _{j \rightarrow \infty} \nu_{j}\left\|\left(H_{f}+N_{f}\right) \Psi\right\| \cdot\left\|\Phi_{\nu_{j}}\right\|=0
$$

Hence $\left\langle H(\mathbf{p}) \Psi, \Phi_{0}\right\rangle=\left\langle\Psi, E(\mathbf{p}) \Phi_{0}\right\rangle$ for all $\Psi \in \mathcal{D}$. Since $\mathcal{D}$ is a core for $\overline{H(\mathbf{p})}$, we have $\Phi_{0} \in \operatorname{Dom}(\overline{H(\mathbf{p})})$ and $\overline{H(\mathbf{p})} \Phi_{0}=E(\mathbf{p}) \Phi_{0}$.

$E_{\nu}(\mathbf{p})$ and $H_{\nu}(\mathbf{p})$ depend on $\mathbf{p}, m, \nu$, etc. When we need to indicate such dependence, we write $E_{\nu}(\mathbf{p}, m, \ldots)$ and $H_{\nu}(\mathbf{p}, m, q, \ldots)$.

In this section, we use the identification

$$
\mathbb{C}^{4} \otimes \mathcal{F}_{\mathrm{rad}}=\bigoplus_{n=0}^{\infty} \mathbb{C}^{4} \otimes \mathcal{F}^{(n)}, \quad \mathcal{F}^{(n)}:=\bigotimes_{\mathrm{sym}}^{n} L^{2}\left(\mathbb{R}_{\mathbf{k}}^{3} \times\{1,2\}\right),
$$

and each vector $\Psi^{(n)} \in \mathbb{C}^{4} \otimes \mathcal{F}^{(n)}$ is identified with a Hilbert space valued function $\Psi^{(n)}(\mathbf{k}, \lambda ; \cdot): \mathbb{R}_{\mathbf{k}}^{3} \times\{1,2\} \rightarrow \mathbb{C}^{4} \otimes \mathcal{F}^{(n-1)}$. For all $(\mathbf{k}, \lambda) \in \mathbb{R}^{3} \times\{1,2\}$, we define a map

$$
a_{\lambda}(\mathbf{k}): \mathbb{C}^{4} \otimes \mathcal{F}_{\text {rad }} \rightarrow \prod_{n=0}^{\infty} \mathbb{C}^{4} \otimes \mathcal{F}^{(n)}:=\left\{\left(\Phi^{(n)}\right)_{n=0}^{\infty} \mid \Phi^{(n)} \in \mathbb{C}^{4} \otimes \mathcal{F}^{(n)}\right\}
$$

by

$$
a_{\lambda}(\mathbf{k}) \Psi:=\left(\Psi^{(1)}(\mathbf{k}, \lambda), \sqrt{2} \Psi^{(2)}(\mathbf{k}, \lambda ; \cdot), \ldots, \sqrt{n} \Psi^{(n)}(\mathbf{k}, \lambda ; \cdot), \ldots\right) \in \prod_{n=0}^{\infty} \mathbb{C}^{4} \otimes \mathcal{F}^{(n)} .
$$

For almost every $(\mathbf{k}, \lambda), a_{\lambda}(\mathbf{k})$ is well-defined as a linear map. The smeared annihilation operator $a(f)$ formally satisfies

$$
a(f) \Psi=\sum_{\lambda=1,2} \int_{\mathbb{R}^{3}} d \mathbf{k} f(\mathbf{k}, \lambda)^{*} a_{\lambda}(\mathbf{k}) \Psi .
$$

It is not necessary to consider $a_{\lambda}(\mathbf{k})$ as an operator valued distribution. This definition of $a_{\lambda}(\mathbf{k})$ is useful for our purpose below (Proposition 6.3). In general, $a_{\lambda}(\mathbf{k}) \Psi \notin \mathbb{C}^{4} \otimes \mathcal{F}_{\text {rad }}$, but one can show that $a_{\lambda}(\mathbf{k}) \Psi \in \mathbb{C}^{4} \otimes \mathcal{F}_{\text {rad }}$ for a class of vectors $\Psi \in \mathbb{C}^{4} \otimes \mathcal{F}_{\text {rad }}$. Let $\mathrm{w}: \mathbb{R}^{3} \rightarrow[0, \infty)$ be an almost positive Borel measurable function. Then, for any $\Psi \in \operatorname{Dom}\left(d \Gamma(\mathrm{w})^{1 / 2}\right)$ and for almost every $(\mathbf{k}, \lambda) \in \mathbb{R}^{3} \times\{1,2\}$, the vector $a_{\lambda}(\mathbf{k}) \Psi$ is a $\mathbb{C}^{4} \otimes \mathcal{F}_{\text {rad-valued function, because, }}$ for any $\Psi \in \operatorname{Dom}\left(d \Gamma(\mathrm{w})^{1 / 2}\right)$, one has

$$
\left\|d \Gamma(\mathrm{w})^{1 / 2} \Psi\right\|^{2}=\sum_{n=1}^{\infty} \sum_{\lambda=1,2} \int_{\mathbb{R}^{3}} d \mathbf{k} \mathrm{w}(\mathbf{k}) n\left\|\Psi^{(n)}(\mathbf{k}, \lambda ; \cdot)\right\|_{\mathbb{C}^{4} \otimes \mathcal{F}(n-1)}^{2}<\infty,
$$

and hence $\sum_{n=1}^{\infty} n\left\|\Psi^{(n)}(\mathbf{k}, \lambda ; \cdot)\right\|_{\mathbb{C}^{4} \otimes \mathcal{F}^{(n-1)}}^{2}<\infty$ for almost every $(\mathbf{k}, \lambda)$. 
We set $\mathbf{g}(\mathbf{k}, \lambda):=\mathbf{g}(\mathbf{k}, \lambda ; 0)$.

Proposition 6.3. Let $\nu>0$. Then $a_{\lambda}(\mathbf{k}) \Phi_{\nu}(\mathbf{p}) \in \operatorname{Dom}\left(H_{\nu}(\mathbf{p})\right)$ and

$$
a_{\lambda}(\mathbf{k}) \Phi_{\nu}(\mathbf{p})=\frac{q}{\sqrt{2}}\left(H_{\nu}(\mathbf{p}-\mathbf{k})-E_{\nu}(\mathbf{p})+\omega_{\nu}(\mathbf{k})\right)^{-1} \boldsymbol{\alpha} \cdot \mathbf{g}(\mathbf{k}, \lambda) \Phi_{\nu}(\mathbf{p})
$$

for almost every $(\mathbf{k}, \lambda) \in \mathbb{R}^{3} \times\{1,2\}$.

Proof. For all $f \in \operatorname{Dom}\left(\omega_{\nu}\right)$ and $\Psi \in \mathcal{D}$, we have

$$
\begin{aligned}
\left\langle\left(H_{\nu}(\mathbf{p})-E_{\nu}(\mathbf{p})\right) \Psi, a(f) \Phi_{\nu}(\mathbf{p})\right\rangle & \\
& =\left\langle\Psi,\left\{-a\left(\omega_{\nu} f\right)+\boldsymbol{\alpha} \cdot a(\mathbf{k} f)+\frac{q}{\sqrt{2}} \boldsymbol{\alpha} \cdot\langle f, \mathbf{g}\rangle\right\} \Phi_{\nu}(\mathbf{p})\right\rangle .
\end{aligned}
$$

Hence

$$
\begin{aligned}
& \sum_{\lambda=1,2} \int_{\mathbb{R}^{3}} d \mathbf{k} f(\mathbf{k}, \lambda)^{*}\left\langle\left(H_{\nu}(\mathbf{p})-E_{\nu}(\mathbf{p})\right) \Psi, a_{\lambda}(\mathbf{k}) \Phi_{\nu}(\mathbf{p})\right\rangle \\
&=\sum_{\lambda=1,2} \int_{\mathbb{R}^{3}} d \mathbf{k} f(\mathbf{k}, \lambda)^{*}\left\langle\Psi,-\omega_{\nu}(\mathbf{k}) a_{\lambda}(\mathbf{k}) \Phi_{\nu}(\mathbf{p})\right. \\
&\left.+\boldsymbol{\alpha} \cdot \mathbf{k} a_{\lambda}(\mathbf{k}) \Phi_{\nu}(\mathbf{p})+\frac{q}{\sqrt{2}} \boldsymbol{\alpha} \cdot \mathbf{g}(\mathbf{k}, \lambda) \Phi_{\nu}(\mathbf{p})\right\rangle .
\end{aligned}
$$

Since $\operatorname{Dom}\left(\omega_{\nu}\right)$ is dense in $L^{2}\left(\mathbb{R}_{\mathbf{k}}^{3} \times\{1,2\}\right)$, we have

$$
\begin{aligned}
& \left\langle\left(H_{\nu}(\mathbf{p})-E_{\nu}(\mathbf{p})\right) \Psi, a_{\lambda}(\mathbf{k}) \Phi_{\nu}(\mathbf{p})\right\rangle \\
& \quad=\left\langle\Psi,\left(-\omega_{\nu}(\mathbf{k}) a_{\lambda}(\mathbf{k})+\boldsymbol{\alpha} \cdot \mathbf{k} a_{\lambda}(\mathbf{k})+\frac{q}{\sqrt{2}} \boldsymbol{\alpha} \cdot \mathbf{g}(\mathbf{k}, \lambda)\right) \Phi_{\nu}(\mathbf{p})\right\rangle
\end{aligned}
$$

for almost every $(\mathbf{k}, \lambda) \in \mathbb{R}^{3} \times\{1,2\}$ and all $\Psi \in \mathcal{D}$. This means that $a_{\lambda}(\mathbf{k}) \Phi_{\nu}(\mathbf{p}) \in$ $D\left(H_{\nu}(\mathbf{p})\right)$ and

$$
\left(H_{\nu}(\mathbf{p})-E_{\nu}(\mathbf{p})+\omega_{\nu}(\mathbf{k})-\boldsymbol{\alpha} \cdot \mathbf{k}\right) a_{\lambda}(\mathbf{k}) \Phi_{\nu}(\mathbf{p})=\frac{q}{\sqrt{2}} \boldsymbol{\alpha} \cdot \mathbf{g}(\mathbf{k}, \lambda) \Phi_{\nu}(\mathbf{p}) .
$$

Hence (6.11) follows.

Lemma 6.4. Suppose that $\hat{\rho}$ is spherically symmetric and $\hat{\rho} \in \operatorname{Dom}\left(\omega^{-3 / 2}\right)$. Assume that $E(\mathbf{p}, m)<E(\mathbf{p}, 0)$. Then

(6.12) $\limsup _{\nu \rightarrow 0}\left\|N_{f}^{1 / 2} \Phi_{\nu}(\mathbf{p})\right\|^{2} \leq \int_{\mathbb{R}^{3}} d \mathbf{k} \frac{q^{2}}{(E(\mathbf{p}-\mathbf{k})-E(\mathbf{p})+|\mathbf{k}|)^{2}} \frac{|\hat{\rho}(\mathbf{k})|^{2}}{|\mathbf{k}|}<\infty$
(6.13) $\limsup _{\nu \rightarrow 0}\left\|H_{f}^{1 / 2} \Phi_{\nu}(\mathbf{p})\right\|^{2} \leq \int_{\mathbb{R}^{3}} d \mathbf{k} \frac{q^{2}}{(E(\mathbf{p}-\mathbf{k})-E(\mathbf{p})+|\mathbf{k}|)^{2}}|\hat{\rho}(\mathbf{k})|^{2}<\infty$ 
Proof. By Proposition 6.3 and (6.10) with $\mathrm{w}=1$, we have

$$
\begin{aligned}
\left\|N_{f}^{1 / 2} \Phi_{\nu}(\mathbf{p})\right\|^{2} & \leq \sum_{\lambda=1}^{2} \int_{\mathbb{R}^{3}} \frac{q^{2}}{2} \frac{\left\|\mathbf{\alpha} \cdot \mathbf{g}(\mathbf{k}, \lambda) \Phi_{\nu}(\mathbf{p})\right\|^{2}}{\left(E_{\nu}(\mathbf{p}-\mathbf{k})-E_{\nu}(\mathbf{p})+|\mathbf{k}|+\nu\right)^{2}} d \mathbf{k} \\
& =\int_{\mathbb{R}^{3}} \frac{q^{2}}{\left(E_{\nu}(\mathbf{p}-\mathbf{k})-E_{\nu}(\mathbf{p})+|\mathbf{k}|+\nu\right)^{2}} \frac{\mid \hat{\rho})\left.\right|^{2}}{|\mathbf{k}|} d \mathbf{k} .
\end{aligned}
$$

By Theorem C.10 and $\hat{\rho} \in \operatorname{Dom}\left(\omega^{-3 / 2}\right)$, the right hand side of (6.12) is finite. Hence, by Proposition C.9 and the Lebesgue convergence theorem, one has (6.12). The proof of (6.13) is similar. The only thing we have to do is set $\mathrm{w}(\mathbf{k})=\omega(\mathbf{k})$.

Proof of Theorem 4.1. By Proposition C.2, we have

$$
0 \leq E(\mathbf{p}-\mathbf{k})-E(\mathbf{p})+|\mathbf{k}| \leq 2|\mathbf{k}|
$$

Hence, by (4.1),

$$
\frac{q^{2}}{4} \int_{\mathbb{R}^{3}} \frac{|\hat{\rho}(\mathbf{k})|^{2}}{|\mathbf{k}|^{3}} d \mathbf{k} \leq \int_{\mathbb{R}^{3}} \frac{q^{2}}{(E(\mathbf{p}-\mathbf{k})-E(\mathbf{p})+|\mathbf{k}|)^{2}} \frac{|\hat{\rho}(\mathbf{k})|^{2}}{|\mathbf{k}|} d \mathbf{k}<1,
$$

which implies $\hat{\rho} \in \operatorname{Dom}\left(\omega^{-3 / 2}\right)$. Hence (6.12) and (6.13) hold.

Since $\Phi_{\nu}(\mathbf{p})$ is a unit vector, there exists a subsequence $\nu_{j}$ such that $\nu_{j} \rightarrow 0$ as $j \rightarrow \infty$ and $\Phi_{0}(\mathbf{p}):=\mathrm{w}_{-} \lim _{j \rightarrow \infty} \Phi_{\nu_{j}}(\mathbf{p})$ exists. Then, by (6.12) and (6.13),

$$
\lim _{j \rightarrow \infty}\left\|N_{f}^{1 / 2} \Phi_{\nu_{j}}\right\|<1, \quad \lim _{j \rightarrow \infty}\left\|H_{f}^{1 / 2} \Phi_{\nu_{j}}\right\|<\infty,
$$

which implies that $\Phi_{0}(\mathbf{p}) \in \operatorname{Dom}\left(N_{f}^{1 / 2}\right) \cap \operatorname{Dom}\left(H_{f}^{1 / 2}\right)$. Hence $\Phi_{0}(\mathbf{p}) \in Q(\overline{H(\mathbf{p})})$, where $Q$ denotes the form domain. For any $\varphi \in \operatorname{Dom}(H(\mathbf{p}))$, we have

$$
\begin{aligned}
\left\langle(H(\mathbf{p})-E(\mathbf{p})) \varphi, \Phi_{0}(\mathbf{p})\right\rangle & =\lim _{j \rightarrow \infty}\left\langle(H(\mathbf{p})-E(\mathbf{p})) \varphi, \Phi_{\nu_{j}}(\mathbf{p})\right\rangle \\
& =\lim _{j \rightarrow \infty}\left\langle\varphi,\left(E_{\nu_{j}}(\mathbf{p})-E(\mathbf{p})-\nu_{j}\left(H_{f}+N_{f}\right)\right) \Phi_{\nu_{j}}(\mathbf{p})\right\rangle=0 .
\end{aligned}
$$

Thus $\Phi_{0}(\mathbf{p}) \in \operatorname{Dom}(\overline{H(\mathbf{p})})$ and $(\overline{H(\mathbf{p})}-E(\mathbf{p})) \Phi_{0}(\mathbf{p})=0$. Therefore, if $\Phi_{0}(\mathbf{p}) \neq 0$, then $\Phi_{0}(\mathbf{p})$ is a ground state of $\overline{H(\mathbf{p})}$. Since $\mathbb{C}^{4}$ is a finite-dimensional space, the vacuum component $\Phi_{\nu_{j}}(\mathbf{p})^{(0)}$ strongly converges to $\Phi_{0}(\mathbf{p})^{(0)}$. Hence

$$
\left\|\Phi_{0}(\mathbf{p})\right\|^{2} \geq\left\|\Phi_{0}(\mathbf{p})^{(0)}\right\|^{2}=\lim _{j \rightarrow \infty}\left\|\Phi_{\nu_{j}}(\mathbf{p})^{(0)}\right\|^{2}=\lim _{j \rightarrow \infty}\left\langle\Phi_{\nu_{j}}(\mathbf{p}), P_{\Omega} \Phi_{\nu_{j}}(\mathbf{p})\right\rangle,
$$

where $P_{\Omega}$ is the orthogonal projection on the vacuum $(1,0,0, \ldots) \in \mathcal{F}_{\text {rad }}$. Thus, using (6.14) and $N_{f} \geq 1-P_{\Omega}$, we have

$$
\left\|\Phi_{0}(\mathbf{p})\right\|^{2} \geq 1-\lim _{j \rightarrow \infty}\left\|N_{f}^{1 / 2} \Phi_{\nu_{j}}(\mathbf{p})\right\|^{2}>0 .
$$

This means that $\Phi_{0}(\mathbf{p}) \neq 0$ and $\Phi_{0}(\mathbf{p})$ is a ground state of $\overline{H(\mathbf{p})}$. 
Proof of Theorem 4.2. Theorem 4.2 follows immediately from Theorems 4.1 and C.10.

Next, we prepare some lemmas for the proof of Theorem 4.4. For a Hilbert space $\mathcal{K}$, we denote by $\mathrm{B}(\mathcal{K})$ the set of all bounded operators on $\mathcal{K}$. The next lemma follows from the second resolvent equation.

Lemma 6.5. Let $\nu>0$. For each $\mathbf{j} \in \mathbb{R}^{3}$ with $|\mathbf{j}|=1$, the operator valued function $\mathbb{R}^{3} \backslash\{\mathbf{0}\}: \mathbf{k} \rightarrow\left(H_{\nu}(\mathbf{p}-\mathbf{k})-E_{\nu}(\mathbf{p})+\omega_{\nu}(\mathbf{k})\right)^{-1} \in \mathrm{B}\left(\mathbb{C}^{4} \otimes \mathcal{F}_{\text {rad }}\right)$ is differentiable in the sense of operator norm, and

$$
\begin{aligned}
& \partial_{\mathbf{j}}\left(H_{\nu}(\mathbf{p}-\mathbf{k})-E_{\nu}(\mathbf{p})+\omega_{\nu}(\mathbf{k})\right)^{-1} \\
& =\left(H_{\nu}(\mathbf{p}-\mathbf{k})-E_{\nu}(\mathbf{p})+\omega_{\nu}(\mathbf{k})\right)^{-1}\left(\boldsymbol{\alpha} \cdot \mathbf{j}-(1+\nu) \frac{\mathbf{k} \cdot \mathbf{j}}{|\mathbf{k}|}\right)\left(H_{\nu}(\mathbf{p}-\mathbf{k})-E_{\nu}(\mathbf{p})+\omega_{\nu}(\mathbf{k})\right)^{-1},
\end{aligned}
$$

where $\partial_{\mathbf{j}}$ means the $\mathbf{j}$-direction derivative.

We fix the following polarization vectors in the rest of this section:

$$
\mathbf{e}^{(1)}(\mathbf{k})=\frac{\left(k_{2},-k_{1}, 0\right)}{\sqrt{k_{1}^{2}+k_{2}^{2}}}, \quad \mathbf{e}^{(2)}(\mathbf{k}):=\frac{\mathbf{k}}{|\mathbf{k}|} \wedge \mathbf{e}^{(1)}(\mathbf{k}) .
$$

Now, recall the definition of the set $S$ (defined in condition $(\Lambda)$ ). We set

$$
\mathrm{X}:=S \backslash\left\{\mathbf{k} \in \mathbb{R}^{3} \mid k_{1}=k_{2}=0\right\}, \quad \mathrm{X}_{R}:=S_{R} \cap \mathrm{X}
$$

By Lemma 6.5 and (6.15), we obtain the following result:

Lemma 6.6. Under the assumptions of Theorem $4.4, a_{\lambda}(\mathbf{k}) \Phi_{\nu}(\mathbf{p})$ is strongly continuously differentiable in $\mathrm{X}$ and

$$
\begin{aligned}
\partial_{j} a_{\lambda}(\mathbf{k}) \Phi_{\nu}(\mathbf{p})= & \frac{q}{\sqrt{2}}\left(H_{\nu}(\mathbf{p}-\mathbf{k})-E_{\nu}(\mathbf{p})+\omega_{\nu}(\mathbf{k})\right)^{-1}\left(\alpha_{j}-(1+\nu) \frac{k_{j}}{|\mathbf{k}|}\right) \\
& \times\left(H_{\nu}(\mathbf{p}-\mathbf{k})-E_{\nu}(\mathbf{p})+\omega_{\nu}(\mathbf{k})\right)^{-1} \boldsymbol{\alpha} \cdot \mathbf{g}(\mathbf{k}, \lambda) \Phi_{\nu}(\mathbf{p}) \\
& +\frac{q}{\sqrt{2}}\left(H_{\nu}(\mathbf{p}-\mathbf{k})-E_{\nu}(\mathbf{p})+\omega_{\nu}(\mathbf{k})\right)^{-1} \boldsymbol{\alpha} \cdot\left(\partial_{j} \mathbf{g}(\mathbf{k}, \lambda)\right) \Phi_{\nu}(\mathbf{p}),
\end{aligned}
$$

where $\partial_{j}$ denotes the strong derivative in $k_{j}(j=1,2,3)$.

We set

$$
\Psi_{j}(\mathbf{k}, \lambda)=\left(\Psi_{j}^{(n)}(\mathbf{k}, \lambda ; \cdot)\right)_{n=0}^{\infty}:=\partial_{j} a_{\lambda}(\mathbf{k}) \Phi_{\nu}(\mathbf{p})
$$


Lemma 6.7. Under the assumptions of Theorem 4.4,

$$
\partial_{j} \Phi_{\nu}^{(n)}(\mathbf{p})\left(\mathbf{k}, \lambda ; X ; k_{2}, \ldots, k_{n}\right)=\frac{1}{\sqrt{n}} \Psi_{j}^{(n-1)}\left(\mathbf{k}, \lambda ; X ; k_{2}, \ldots, k_{n}\right), \quad k_{\ell}=\left(\mathbf{k}_{\ell}, \lambda_{\ell}\right),
$$

for all $X \in\{1,2,3,4\}, \mathbf{k}, \mathbf{k}_{\ell} \in \mathbf{X}, n \in \mathbb{N}, \lambda, \lambda_{\ell}=1,2$ and $j=1,2,3$, where $\partial_{j}$ is the distributional derivative with respect to $k_{j}$.

Note that the $\partial_{j}$ on the left hand side is a distributional derivative and the one in $\Psi_{j}$ is a strong derivative.

Proof. In this proof, for simplicity, we do not indicate $X, \lambda, \lambda_{\ell}$ and $\mathbf{p}$. The operator $\delta_{h}$ is defined by $\delta_{h} f(\mathbf{k}):=f(\mathbf{k}+h \mathbf{j})-f(\mathbf{k})$ for all functions $f(\mathbf{k})$. Let $\psi\left(\mathbf{k}, \mathbf{k}_{2}, \ldots, \mathbf{k}_{n}\right) \in C_{0}^{\infty}\left(\mathbf{X}^{n+1}\right)$ be arbitrary. Clearly, we have $\left(\partial_{j} \psi\right)(\mathbf{k}, K)=$ $\lim _{h \rightarrow 0} h^{-1}(\psi(\mathbf{k}+h \mathbf{j}, K)-\psi(\mathbf{k}, K))$ uniformly, where $K=\left(\mathbf{k}_{2}, \ldots, \mathbf{k}_{n}\right)$ and $\mathbf{j}$ is the unit vector of the $j$-th axis. By the definition of the distributional derivative, we have

$$
\begin{aligned}
\int_{\mathbb{R}^{3 n}} d \mathbf{k} d K \psi(\mathbf{k}, K) \partial_{j} \Phi_{\nu}^{(n)}(\mathbf{k}, K) & =-\int_{\mathbb{R}^{3 n}} d \mathbf{k} d K\left(\partial_{j} \psi\right)(\mathbf{k}, K) \Phi_{\nu}^{(n)}(\mathbf{k}, K) \\
& =-\lim _{h \rightarrow 0} \int_{\mathbb{R}^{3 n}} d \mathbf{k} d K \frac{1}{-h}\left(\delta_{-h} \psi\right)(\mathbf{k}, K) \Phi_{\nu}^{(n)}(\mathbf{k}, K) \\
& =\lim _{h \rightarrow 0} \int_{\mathbb{R}^{3 n}} d \mathbf{k} d K \psi(\mathbf{k}, K) \frac{1}{h}\left(\delta_{h} \Phi_{\nu}^{(n)}\right)(\mathbf{k}, K) .
\end{aligned}
$$

By Schwarz' inequality, we have

$$
\begin{array}{r}
\left|\int_{\mathbb{R}^{3}} d \mathbf{k}\left[\int_{\mathbb{R}^{3(n-1)}} d K \psi(\mathbf{k}, K)\left\{\frac{1}{h}\left[\Phi_{\nu}^{(n)}(\mathbf{k}+h \mathbf{j}, K)-\Phi_{\nu}^{(n)}(\mathbf{k}, K)\right]-\frac{1}{\sqrt{n}} \Psi^{(n-1)}(\mathbf{k}, K)\right\}\right]\right| \\
\leq \int_{\mathbb{R}^{3}} d \mathbf{k}\|\psi(\mathbf{k}, \cdot)\|_{L^{2}\left(\mathbb{R}^{3(n-1)}\right)}\left\|\frac{\delta_{h}}{h} \Phi_{\nu}^{(n)}(\mathbf{k}, \cdot)-\frac{1}{\sqrt{n}} \Psi^{(n-1)}(\mathbf{k}, \cdot)\right\|_{L^{2}\left(\mathbb{R}^{3(n-1)}\right)}
\end{array}
$$

Note that, for all $\mathbf{k} \in \mathrm{X}, h^{-1} \delta_{h} \Phi_{\nu}^{(n)}(\mathbf{k}, \cdot)$ strongly converges to $\frac{1}{\sqrt{n}} \Psi^{(n-1)}(\mathbf{k}, \cdot)$ in $L^{2}\left(\mathrm{X}^{3(n-1)}\right)$ by Lemma 6.6. Moreover, by Lemma 6.6 and the assumption that $\hat{\rho}$ is continuously differentiable, the function $\mathbf{k} \mapsto \Psi^{(n-1)}(\mathbf{k}, \cdot)$ is strongly continuous in $\mathbf{X}$. Let $D$ be the closure of $\left\{\mathbf{k} \in \mathbb{R}^{3} \mid\|\psi(\mathbf{k}, \cdot)\|_{L^{2}\left(\mathbb{R}^{3(n-1)}\right)} \neq 0\right\}$. Note that $D \subset \mathbf{X}$ is a compact set and $d:=\operatorname{dist}\left(D, X^{\mathrm{c}}\right)>0$.

For every $\mathbf{k} \in D$ and $h$ with $|h|<d$, we have

$$
\frac{\delta_{h}}{h} \Phi_{\nu}^{(n)}(\mathbf{k}, \cdot)=\mathrm{s}-\int_{0}^{1} \frac{1}{\sqrt{n}} \Psi^{(n-1)}(\mathbf{k}+t h \mathbf{j}, \cdot) d t,
$$

where s- $\int$ means the strong integral in $L^{2}\left(\mathrm{X}^{3(n-1)}\right)$. Since $\left\|\Psi^{(n-1)}(\mathbf{k}, \cdot)\right\|_{L^{2}\left(\mathbb{R}^{3(n-1)}\right)}$ is continuous in $\mathbf{k} \in \mathbf{X}$, it is bounded on the compact set $D$. For any $\mathbf{k} \in D$ and 
$|h|<d$, we have

$$
\begin{aligned}
& \left\|\frac{\delta_{h}}{|h|} \Phi_{\nu}^{(n)}(\mathbf{k}, \cdot)-\frac{1}{\sqrt{n}} \Psi^{(n-1)}(\mathbf{k}, \cdot)\right\|_{L^{2}\left(\mathbb{R}^{3(n-1)}\right)} \\
& \leq \sup _{|t| \leq 1} \frac{1}{\sqrt{n}}\left\|\Psi^{(n-1)}(\mathbf{k}+t h \mathbf{j}, \cdot)\right\|_{L^{2}\left(\mathbb{R}^{3(n-1)}\right)}+\frac{1}{\sqrt{n}}\left\|\Psi^{(n-1)}(\mathbf{k}, \cdot)\right\|_{L^{2}\left(\mathbb{R}^{3(n-1)}\right)} \leq \mathrm{const},
\end{aligned}
$$

where "const" means a constant independent of $\mathbf{k}$ and $h$. Applying the Lebesgue dominated convergence theorem, we can see that the right hand side of (6.16) converges to zero as $|h| \rightarrow 0$.

By Lemmas 6.5-6.6 and direct calculations, we obtain the following inequality.

Lemma 6.8. Under the assumptions of Theorem 4.4,

$$
\begin{aligned}
\left\|\partial_{j} a_{\lambda}(\mathbf{k}) \Phi_{\nu}(\mathbf{p})\right\| \leq & \frac{|q|}{\sqrt{2}}(2+\nu)\left(E_{\nu}(\mathbf{p}-\mathbf{k})-E_{\nu}(\mathbf{p})+\omega_{\nu}(\mathbf{k})\right)^{-2} \frac{|\hat{\rho}(\mathbf{k})|}{|\mathbf{k}|^{1 / 2}} \\
& +\frac{|q|}{\sqrt{2}}\left(E_{\nu}(\mathbf{p}-\mathbf{k})-E_{\nu}(\mathbf{p})+\omega_{\nu}(\mathbf{k})\right)^{-1} \frac{\left|\partial_{j} \hat{\rho}(\mathbf{k})\right|}{|\mathbf{k}|^{1 / 2}} \\
& +\frac{|q|}{\sqrt{2}}\left(E_{\nu}(\mathbf{p}-\mathbf{k})-E_{\nu}(\mathbf{p})+\omega_{\nu}(\mathbf{k})\right)^{-1} \frac{|\hat{\rho}(\mathbf{k})|}{|\mathbf{k}|^{3 / 2}} \\
& +\frac{|q|}{\sqrt{2}}\left(E_{\nu}(\mathbf{p}-\mathbf{k})-E_{\nu}(\mathbf{p})+\omega_{\nu}(\mathbf{k})\right)^{-1} \frac{|\hat{\rho}(\mathbf{k})|}{|\mathbf{k}|^{1 / 2}}\left|\partial_{j} \mathbf{e}^{(\lambda)}(\mathbf{k})\right|
\end{aligned}
$$

for all $\mathbf{k} \in \mathbf{X}, \lambda=1,2, j=1,2,3$.

Our polarization vectors (6.15) satisfy

$$
\left|\partial_{j} \mathbf{e}^{(\lambda)}(\mathbf{k})\right| \leq \frac{2}{\sqrt{k_{1}^{2}+k_{2}^{2}}} \quad \text { for } \mathbf{k} \in \mathbb{R}^{3} \backslash\left\{\mathbf{k}^{\prime} \in \mathbb{R}^{3} \mid k_{1}^{\prime}=k_{2}^{\prime}=0\right\} .
$$

We set

$$
\begin{aligned}
& f_{\nu}^{(1)}(\mathbf{k}):=\left(E_{\nu}(\mathbf{p}-\mathbf{k})-E_{\nu}(\mathbf{p})+\omega_{\nu}(\mathbf{k})\right)^{-2} \frac{|\hat{\rho}(\mathbf{k})|}{|\mathbf{k}|^{1 / 2}}, \\
& f_{\nu}^{(2)}(\mathbf{k}):=\left(E_{\nu}(\mathbf{p}-\mathbf{k})-E_{\nu}(\mathbf{p})+\omega_{\nu}(\mathbf{k})\right)^{-1} \frac{\left|\partial_{j} \hat{\rho}(\mathbf{k})\right|}{|\mathbf{k}|^{1 / 2}} \\
& f_{\nu}^{(3)}(\mathbf{k}):=\left(E_{\nu}(\mathbf{p}-\mathbf{k})-E_{\nu}(\mathbf{p})+\omega_{\nu}(\mathbf{k})\right)^{-1} \frac{|\hat{\rho}(\mathbf{k})|}{|\mathbf{k}|^{3 / 2}}, \\
& f_{\nu}^{(4)}(\mathbf{k}):=\left(E_{\nu}(\mathbf{p}-\mathbf{k})-E_{\nu}(\mathbf{p})+\omega_{\nu}(\mathbf{k})\right)^{-1} \frac{|\hat{\rho}(\mathbf{k})|}{|\mathbf{k}|^{1 / 2}}\left|\partial_{j} \mathbf{e}^{(\lambda)}(\mathbf{k})\right| .
\end{aligned}
$$

Lemma 6.9. Under the assumptions of Theorem 4.4,

$$
\sup _{0<\nu \leq 1}\left\|f_{\nu}^{(j)}\right\|_{L^{p}\left(S_{R}\right)}<\infty, \quad j=1,2,3,4, p \in[1,2) .
$$


Proof. First we consider the case $\mathbf{p} \neq \mathbf{0}$. Let $b_{\nu}(\mathbf{p})$ be the constant defined in Theorem C.10. Since $b_{\nu}(\mathbf{p})$ is continuous in $\nu$ for fixed $\mathbf{p}$, Theorem C.10 guarantees $\sup _{0 \leq \nu \leq 1} b_{\nu}(\mathbf{p})=\max _{0 \leq \nu \leq 1} b_{\nu}(\mathbf{p})<1$. By Theorem C.10, we have

$$
\left(E_{\nu}(\mathbf{p}-\mathbf{k})-E_{\nu}(\mathbf{p})+|\mathbf{k}|\right)^{-1} \leq \frac{1}{1-b_{\nu}(\mathbf{p})} \max \left\{\frac{1}{|\mathbf{k}|}, \frac{1}{|\mathbf{p}|}\right\} \leq C \max \left\{\frac{1}{|\mathbf{k}|}, \frac{1}{|\mathbf{p}|}\right\}
$$

where

$$
C:=\sup _{0<\nu \leq 1} \frac{1}{1-b_{\nu}(\mathbf{p})}
$$

is a finite constant. Hence

$$
f_{\nu}^{(1)}(\mathbf{k}) \leq C^{2}\left\{\frac{1}{|\mathbf{k}|^{2}}+\frac{1}{|\mathbf{p}|^{2}}\right\} \frac{|\hat{\rho}(\mathbf{k})|}{|\mathbf{k}|^{1 / 2}} .
$$

Since $S_{R}$ is a bounded region, by the assumption $|\mathbf{k}|^{-5 / 2}|\hat{\rho}(\mathbf{k})| \in L^{p}\left(S_{R}\right)$, we obtain

$$
\sup _{0<\nu \leq 1}\left\|f_{\nu}^{(1)}\right\|_{L^{p}\left(S_{R}\right)}<\infty
$$

Similarly,

$$
\sup _{0<\nu \leq 1}\left\|f_{\nu}^{(j)}\right\|_{L^{2}\left(S_{R}\right)}<\infty, \quad j=2,3
$$

By (6.17), we have

$$
f_{\nu}^{(4)}(\mathbf{k}) \leq C^{2}\left\{\frac{1}{|\mathbf{k}|}+\frac{1}{|\mathbf{p}|}\right\} \frac{1}{\sqrt{k_{1}^{2}+k_{2}^{2}}} \frac{|\hat{\rho}(\mathbf{k})|}{|\mathbf{k}|^{1 / 2}} .
$$

By using polar coordinates, we have

$$
\begin{aligned}
\int_{S_{R}} f_{\nu}^{(4)}(\mathbf{k}) d \mathbf{k} & \leq 2 \pi C \int_{[0, \pi)} \sin \theta d \theta\left[\frac{1}{\sin \theta}\right]^{p} \int_{[0, R)}|\mathbf{k}|^{2-p}\left(\frac{|\mathbf{k}|+|\mathbf{p}|}{|\mathbf{k}| \cdot|\mathbf{p}|}\right)^{p} \frac{|\hat{\rho}(\mathbf{k})|^{p}}{|\mathbf{k}|} d|\mathbf{k}| \\
& <\infty
\end{aligned}
$$

Next we consider the case $\mathbf{p}=0$. By (C.4), we have

$$
\left(E_{\nu}(-\mathbf{k})-E_{\nu}(\mathbf{0})+\omega_{\nu}(\mathbf{k})\right)^{-1} \leq \begin{cases}\frac{P}{a_{\nu}(P)|\mathbf{k}|} & \text { if }|\mathbf{k}| \leq P \\ a_{\nu}(P)^{-1} & \text { if }|\mathbf{k}|>P\end{cases}
$$

for any $P>0$. By similar arguments, one can prove (6.18).

Let $W^{1, p}(\mathcal{X})$ be the Sobolev space on the configuration space $\mathcal{X}$, i.e., the set of all $L^{p}$-functions with their first derivatives also in $L^{p}$. 
Lemma 6.10. Under the assumptions of Theorem 4.4, the $n$-th component of the massive ground state satisfies $\Phi_{\nu}^{(n)} \in \bigoplus^{4} W^{1, p}\left(\left(\mathrm{X}_{R} \times\{1,2\}\right)^{n}\right)$ for all $p \in[1,2)$ and all $R>0$, and

$$
\sup _{0<\nu<1}\left\|\Phi_{\nu}^{(n)}(\mathbf{p})\right\|_{\oplus^{4} W^{1, p}\left(\left(\mathrm{X}_{R} \times\{1,2\}\right)^{n}\right)}<\infty .
$$

Proof. By Lemma 6.7, we have

$$
\begin{aligned}
&\left(\nabla_{\mathbf{k}} a_{\lambda}(\mathbf{k}) \Phi_{\nu}(\mathbf{p})\right)^{(n-1)}\left(X ; \mathbf{k}_{1}, \lambda_{1} ; \ldots ; \mathbf{k}_{n-1}, \lambda_{n-1}\right) \\
& \quad=\sqrt{n} \nabla_{\mathbf{k}} \Phi_{\nu}^{(n)}\left(\mathbf{p} ; X ; \mathbf{k}, \lambda ; \mathbf{k}_{1}, \lambda_{1} ; \ldots ; \mathbf{k}_{n-1}, \lambda_{n-1}\right) .
\end{aligned}
$$

Using Hölder's inequality and making a change of variables, one has, for all $p<2$,

$$
\begin{array}{r}
\sum_{X=1}^{4} \sum_{\lambda_{1}, \ldots, \lambda_{n} \in\{1,2\}} \int_{\left(\mathbf{X}_{R}\right)^{n}} d \mathbf{k}_{1} \cdots d \mathbf{k}_{n} \sum_{i=1}^{n}\left|\nabla_{\mathbf{k}_{i}} \Phi_{\nu}^{(n)}\left(\mathbf{p} ; X ; \mathbf{k}_{1}, \lambda_{1} ; \ldots ; \mathbf{k}_{n}, \lambda_{n}\right)\right|^{p} \\
\leq C \int_{\mathbf{X}_{R}} d \mathbf{k}\left\|\nabla_{\mathbf{k}} a_{\lambda}(\mathbf{k}) \Phi_{\nu}(\mathbf{p})\right\|^{p},
\end{array}
$$

where $C$ is a constant independent of $\nu$. By Lemmas 6.8 and 6.9, the right hand side of (6.19) is finite uniformly in $\nu>0$.

Proof of Theorem 4.4. As shown in the proof of Theorem 4.1, there exists a sequence $\left\{\nu_{j}\right\}_{j=1}^{\infty}$ such that the limit $\Phi_{0}(\mathbf{p}):=\mathrm{w}_{-}-\lim _{j \rightarrow \infty} \Phi_{\nu_{j}}(\mathbf{p})$ exists, and $\Phi_{0}(\mathbf{p}) \in$ $\operatorname{Dom}\left(H_{f}^{1 / 2}\right) \cap \operatorname{Dom}\left(N_{f}^{1 / 2}\right)$. Thus, $\Phi_{0} \in Q(H(\mathbf{p}))$. If $\Phi_{0}(\mathbf{p}) \neq 0$, then $\Phi_{0}(\mathbf{p})$ is a ground state of $H(\mathbf{p})$. In the following, we show that indeed $\Phi_{0}(\mathbf{p}) \neq 0$.

Any vector $\Psi \in \bigoplus^{4} \mathcal{F}^{n}=\mathbb{C}^{4} \otimes \mathcal{F}^{n}$ is a function of the particle helicity $X \in\{1,2,3,4\}$, the $n$-photon wave number $\left(\mathbf{k}_{1}, \ldots, \mathbf{k}_{n}\right) \in \mathbb{R}^{3 n}$, and the photon polarization $\lambda_{1}, \ldots, \lambda_{n} \in\{1,2\}$. For simplicity, we set

$$
\begin{aligned}
& \Phi_{j}^{(n)}\left(\mathbf{k}_{1}, \ldots, \mathbf{k}_{n}\right):=\Phi_{\nu_{j}}(\mathbf{p})^{(n)}\left(X ; \mathbf{k}_{1}, \lambda_{1} ; \ldots ; \mathbf{k}_{n}, \lambda_{n}\right), \\
& \Phi_{0}^{(n)}\left(\mathbf{k}_{1}, \ldots, \mathbf{k}_{n}\right):=\Phi_{0}(\mathbf{p})^{(n)}\left(X ; \mathbf{k}_{1}, \lambda_{1} ; \ldots ; \mathbf{k}_{n}, \lambda_{n}\right)
\end{aligned}
$$

for $X \in\{1,2,3,4\}$ and $\lambda_{1}, \ldots, \lambda_{n} \in\{1,2\}$. Note that $\Phi_{j}^{(n)}, \Phi_{0}^{(n)} \in L^{2}\left(\mathbb{R}^{3 n}\right)$. We show that $\operatorname{sim}_{j \rightarrow \infty} \Phi_{j}^{(n)}=\Phi_{0}^{(n)}$ for all $n \in \mathbb{N}, X \in\{1,2,3,4\}$ and $\lambda_{1}, \ldots, \lambda_{n} \in$ $\{1,2\}$.

By Lemma 6.10 and the Rellich-Kondrashov theorem,

$$
\lim _{j \rightarrow \infty}\left\|\Phi_{j}^{(n)}-\Phi_{0}^{(n)}\right\|_{L^{2}\left(\mathbf{X}_{R}^{n}\right)}=0
$$

for all $R>0$ (see [GLL, p. 578] for details). We set $\Phi_{j}:=\left(\Phi_{j}^{(n)}\right)_{n=0}^{\infty}$ and $\Phi_{0}:=$ $\left(\Phi_{0}^{(n)}\right)_{n=0}^{\infty} \in \bigoplus^{4} \mathcal{F}_{\text {rad }}$. Let $\chi_{R}$ be the characteristic function of the ball $\left\{\mathbf{k} \in \mathbb{R}^{3} \mid\right.$ 
$|\mathbf{k}|<R\}$. We denote the orthogonal projection onto $\bigoplus_{j=0}^{n} \mathbb{C}^{4} \otimes \mathcal{F}^{j}$ by $P_{n}$. Then we have

$$
\begin{aligned}
\left\|\Gamma\left(\chi_{R}\right)\left(\Phi_{j}-\Phi_{0}\right)\right\|^{2} & =\left\|P_{n} \Gamma\left(\chi_{R}\right)\left(\Phi_{j}-\Phi_{0}\right)\right\|^{2}+\left\|\left(1-P_{n}\right) \Gamma\left(\chi_{R}\right)\left(\Phi_{j}-\Phi_{0}\right)\right\|^{2} \\
& \leq\left\|P_{n} \Gamma\left(\chi_{R}\right)\left(\Phi_{j}-\Phi_{0}\right)\right\|^{2}+\frac{1}{n}\left\|N_{f}^{1 / 2} \Gamma\left(\chi_{R}\right)\left(\Phi_{j}-\Phi_{0}\right)\right\|^{2}
\end{aligned}
$$

Since each component $\left(\Gamma\left(\chi_{R}\right) \Phi_{j}\right)^{(n)}$ converges to $\left(\Gamma\left(\chi_{R}\right) \Phi_{0}\right)^{(n)}$ strongly as $j \rightarrow \infty$, we have

$$
\limsup _{j \rightarrow \infty}\left\|\Gamma\left(\chi_{R}\right)\left(\Phi_{j}-\Phi_{0}\right)\right\|^{2} \leq \frac{1}{n} \limsup _{j \rightarrow \infty}\left\|N_{f}^{1 / 2}\left(\Phi_{j}-\Phi_{0}\right)\right\|^{2}
$$

for all $n \in \mathbb{N}$. By Lemma $6.4, \lim \sup _{j \rightarrow \infty}\left\|N_{f}^{1 / 2}\left(\Phi_{j}-\Phi_{0}\right)\right\|^{2}<\infty$. Thus we obtain

$$
\operatorname{s}_{j \rightarrow \infty} \Gamma\left(\chi_{R}\right) \Phi_{j}=\Gamma\left(\chi_{R}\right) \Phi_{0} .
$$

Therefore for all $R>0$ we have

$$
\begin{aligned}
\left\|\Phi_{j}-\Phi_{0}\right\| & =\left\|\Gamma\left(\chi_{R}\right)\left(\Phi_{j}-\Phi_{0}\right)\right\|+\left\|\left(1-P_{0}\right)\left(\Gamma\left(\chi_{R}\right)-1\right)\left(\Phi_{j}-\Phi_{0}\right)\right\|^{2} \\
& \leq\left\|\Gamma\left(\chi_{R}\right)\left(\Phi_{j}-\Phi_{0}\right)\right\|+\left\|\left(1-P_{0}\right)\left(1-\Gamma\left(\chi_{R}\right)\right) H_{f}^{-1 / 2}\right\| \cdot\left\|H_{f}^{1 / 2}\left(\Phi_{j}-\Phi_{0}\right)\right\| \\
& \leq\left\|\Gamma\left(\chi_{R}\right)\left(\Phi_{j}-\Phi_{0}\right)\right\|+\frac{C}{R^{1 / 2}},
\end{aligned}
$$

where $C$ is a constant independent of $R>0$. By (6.21), we obtain

$$
\underset{j \rightarrow \infty}{\mathrm{s}-\lim _{j}} \Phi_{j}=\Phi_{0},
$$

which implies that $\Phi_{0}$ is a normalized ground state of $\overline{H(\mathbf{p})}$.

\section{$\S 7$. Proof of Theorem 5.2}

Throughout this section we assume that the assumptions of Theorem 5.2 hold. By Appendices $\mathrm{A}$ and $\mathrm{B}$, it suffices to prove Theorem 5.2 in the case $\mathbf{e}=\overline{\mathbf{e}}$. Here $\overline{\mathbf{e}}$ is the polarization vector defined in (B.1). Note that $\overline{\mathbf{e}}$ depends on $\mathbf{j}$. By assumption, there exists a non-negative constant $t$ such that $\mathbf{p}=t \mathbf{j}$. We choose a matrix $T \in S O(3)$ such that $T^{-1} \mathbf{p}=(0,0,|\mathbf{p}|)$ and $T^{-1} \mathbf{j}=(0,0,1)$. Let $U$ be the unitary operator defined in the proof of Proposition C.4. By (C.1), we obtain

$$
U \overline{H(\mathbf{p})} U^{*}=\overline{\left(|\mathbf{p}| \alpha_{3}+m \beta+H_{f}-\boldsymbol{\alpha} \cdot d \Gamma(\mathbf{k})-q \boldsymbol{\alpha} \cdot \Phi_{\mathrm{S}}(\vec{\lambda})\right)},
$$

where

$$
\vec{\lambda}=\left(\lambda_{1}, \lambda_{2}, \lambda_{3}\right)=\frac{\hat{\rho}(T \mathbf{k})}{|\mathbf{k}|^{1 / 2}}\left(T^{-1} \overline{\mathbf{e}}^{(1)}(T \mathbf{k}), T^{-1} \overline{\mathbf{e}}^{(2)}(T \mathbf{k})\right) \in\left(L^{2}\left(\mathbb{R}_{\mathbf{k}}^{3} \times\{1,2\}\right)\right)^{3} .
$$


Since $T \in S O(3)$, we have

$$
\begin{aligned}
& T^{-1} \overline{\mathbf{e}}^{(1)}(T \mathbf{k})=\frac{T^{-1}[(T \mathbf{k}) \wedge \mathbf{j}]}{|(T \mathbf{k}) \wedge \mathbf{j}|}=\frac{\mathbf{k} \wedge(0,0,1)}{|\mathbf{k} \wedge(0,0,1)|}, \\
& T^{-1} \overline{\mathbf{e}}^{(2)}(T \mathbf{k})=\frac{\mathbf{k}}{|\mathbf{k}|} \wedge\left(T^{-1} \overline{\mathbf{e}}^{(1)}(T \mathbf{k})\right) .
\end{aligned}
$$

It is easy to see that $\hat{\rho}\left(T R^{\prime} \mathbf{k}\right)=\hat{\rho}(T \mathbf{k}), \mathbf{k} \in \mathbb{R}^{3}$, for all $R^{\prime} \in O(3)$ such that $R^{\prime}(0,0,1)=(0,0,1)$. Since $\mathbf{S}=(i / 4) \boldsymbol{\alpha} \wedge \boldsymbol{\alpha}$, we have

$$
U(\mathbf{j} \cdot \mathbf{S}) U^{*}=\frac{i}{4} \mathbf{j} \cdot[(T \boldsymbol{\alpha}) \cdot(T \boldsymbol{\alpha})]=\frac{i}{4} \mathbf{j} \cdot[T(\boldsymbol{\alpha} \wedge \boldsymbol{\alpha})]=\frac{i}{4}(\boldsymbol{\alpha} \wedge \boldsymbol{\alpha})_{3}=S_{3} .
$$

Moreover, one can show that $U(\mathbf{j} \cdot d \Gamma(\vec{\ell})) U^{*}=d \Gamma\left(\ell_{3}\right)$. Therefore,

$$
U J_{\mathbf{j}}(\overline{\mathbf{e}}) U^{*}=S_{3}+d \Gamma\left(\ell_{3}\right),
$$

and hence it is sufficient to prove Theorem 5.2 in the case

$$
\mathbf{p}=(0,0,|\mathbf{p}|), \quad \mathbf{j}=(0,0,1) .
$$

Proof of Theorem 5.2. We assume (7.1) holds. We put

$$
\check{\mathbf{e}}^{(1)}(\mathbf{k}):=\frac{\left(k_{2},-k_{1}, 0\right)}{\sqrt{k_{1}^{2}+k_{2}^{2}}}, \quad \check{\mathbf{e}}^{(2)}(\mathbf{k}):=\frac{\mathbf{k}}{|\mathbf{k}|} \wedge \check{\mathbf{e}}^{(1)}(\mathbf{k}) .
$$

For a real parameter $\theta \in \mathbb{R}$, we set

$$
W:=\exp \left[i \theta J_{\mathbf{j}}(\check{\mathbf{e}})\right], \quad \Theta:=\left[\begin{array}{ccc}
\cos \theta & -\sin \theta & 0 \\
\sin \theta & \cos \theta & 0 \\
0 & 0 & 1
\end{array}\right] .
$$

Then we obtain

$$
\begin{aligned}
W \boldsymbol{\alpha} W^{*} & =\Theta \boldsymbol{\alpha}, \quad W \beta W^{*}=\beta, \\
W d \Gamma(\mathbf{k}) W^{*} & =\Theta d \Gamma(\mathbf{k}), \quad W H_{f}(m) W^{*}=H_{f}(m), \\
W \mathbf{A} W^{*} & =\Theta \mathbf{A} .
\end{aligned}
$$

Here, to show (7.4), we used the specific form of è:

$$
\check{\mathbf{e}}^{(\lambda)}(\Theta \mathbf{k})=\Theta \check{\mathbf{e}}^{(\lambda)}(\mathbf{k}), \quad \lambda=1,2 .
$$

Since $\theta \in \mathbb{R}$ is arbitrary, (7.2)-(7.4) imply that $\overline{H(\mathbf{p})}$ strongly commutes with $J_{\mathbf{j}}(\check{\mathbf{e}})$. Thus, $\overline{H(\mathbf{p})}$ is reduced by the projection onto the eigenspace of $J_{\mathbf{j}}(\check{\mathbf{e}})$. In other words, $\overline{H(\mathbf{p})}$ decomposes as

$$
\overline{H(\mathbf{p})} \cong \bigoplus_{z \in \mathbb{Z}_{1 / 2}} H(\mathbf{p}: z)
$$


in the sense of (5.1). We furthermore define unitary operators $\eta, \tau$ and $\Upsilon$ by

$$
\begin{aligned}
(\eta f)(\mathbf{k}, \lambda) & := \begin{cases}-f\left(k_{1},-k_{2}, k_{3}, 1\right) & \text { if } \lambda=1, \\
f\left(k_{1},-k_{2}, k_{3}, 2\right) & \text { if } \lambda=2, f \in L^{2}\left(\mathbb{R}_{\mathbf{k}}^{3} \times\{1,2\}\right),\end{cases} \\
\tau & :=\alpha_{1} \alpha_{2} \beta, \quad \Upsilon:=\tau \cdot \Gamma(\eta) .
\end{aligned}
$$

It is easy to see that

$$
\begin{aligned}
\eta \ell_{3} \eta^{*} & =-\ell_{3}, \quad \tau S_{3} \tau^{*}=-S_{3}, \\
\eta k_{1} \eta^{*} & =k_{1}, \quad \eta k_{2} \eta^{*}=-k_{2}, \quad \eta k_{3} \eta^{*}=k_{3}, \\
\tau \alpha_{1} \tau^{*} & =\alpha_{1}, \quad \tau \alpha_{2} \tau^{*}=-\alpha_{2}, \quad \tau \alpha_{3} \tau^{*}=\alpha_{3}, \quad \tau \beta \tau^{*}=\beta, \\
\eta \check{\mathbf{e}}^{(1)}(\mathbf{k}) \eta^{-1} & =\frac{\left(k_{2},-\left(-k_{1}\right), 0\right)}{\sqrt{k_{1}^{2}+k_{2}^{2}}}, \quad \eta \check{\mathbf{e}}^{(2)}(\mathbf{k}) \eta^{-1}=\frac{\left(k_{1} k_{3},-k_{2} k_{3},-k_{1}^{2}-k_{2}^{2}\right)}{|\mathbf{k}| \sqrt{k_{1}^{2}+k_{2}^{2}}} .
\end{aligned}
$$

Hence

$$
\Upsilon \overline{H(\mathbf{p})} \Upsilon^{*}=\overline{H(\mathbf{p})}, \quad \Upsilon J_{\mathbf{j}} \Upsilon^{*}=-J_{\mathbf{j}}
$$

Let $E(z), z \in \mathbb{Z}_{1 / 2}$, be the orthogonal projection onto $\operatorname{ker}\left(J_{\mathbf{j}}-z\right)$. Note that $\operatorname{Ran}(E(z))=\mathcal{F}(z)$. Moreover $E(-z) \Upsilon E(z)$ is a unitary operator from $\operatorname{Ran}(E(z))$ to $\operatorname{Ran}(E(-z))$ and

$$
\begin{aligned}
E(-z) \Upsilon E(z) H(\mathbf{p}: z) E(z) \Upsilon^{*} E(-z) & =E(-z) \Upsilon E(z) \Upsilon^{*} \overline{H(\mathbf{p})} \Upsilon E(z) \Upsilon^{*} E(-z) \\
& =H(\mathbf{p}:-z)
\end{aligned}
$$

Therefore $H(\mathbf{p}: z)$ is unitarily equivalent to $H(\mathbf{p}:-z)$ for all $z \in \mathbb{Z}_{1 / 2}$.

\section{Appendix A. Remarks on polarization vectors}

In this appendix, we show that quantum electrodynamics is independent of the choice of polarization vectors, i.e., the Hamiltonians defined by different polarization vectors are unitarily equivalent. We show the equivalence only for the Hamiltonians $H$ and $H(\mathbf{p})$, but one can apply our proof to the Pauli-Fierz model and various QED models. The proof here is independent of the choice of $\hat{\rho}$ and $\omega$.

We assume that the polarization vectors $\mathbf{e}^{(1)}(\mathbf{k}), \mathbf{e}^{(2)}(\mathbf{k})$ and $\mathbf{k}$ form a righthanded system:

$$
\mathbf{k} \cdot \mathbf{e}^{(1)}(\mathbf{k})=0, \quad\left\|\mathbf{e}^{(1)}(\mathbf{k})\right\|_{\mathbb{R}^{3}}=1, \quad \mathbf{e}^{(2)}(\mathbf{k})=\frac{\mathbf{k}}{|\mathbf{k}|} \wedge \mathbf{e}^{(1)}(\mathbf{k}), \quad \mathbf{k} \in \mathbb{R}^{3}
$$

Next, we take any polarization vectors $\mathbf{e}^{\prime(1)}, \mathbf{e}^{\prime(2)}$ :

$$
\mathbf{k} \cdot \mathbf{e}^{\prime(\lambda)}(\mathbf{k})=0, \quad \mathbf{e}^{\prime(\lambda)}(\mathbf{k}) \cdot \mathbf{e}^{\prime(\mu)}(\mathbf{k})=\delta_{\lambda, \mu}, \quad \mathbf{k} \in \mathbb{R}^{3}, \lambda, \mu \in\{1,2\} .
$$


Let $H^{\prime}$ and $H^{\prime}(\mathbf{p})$ be the Hamiltonians $H$ and $H(\mathbf{p})$ with $\mathbf{e}^{(\lambda)}$ replaced by $\mathbf{e}^{\prime(\lambda)}$, $\lambda=1,2$, respectively.

Theorem A.1. Assume that $H$ is essentially self-adjoint. Then $H^{\prime}$ is also essentially self-adjoint and $\bar{H}$ is unitarily equivalent to $\bar{H}^{\prime}$ by means of a unitary operator $U\left(\mathbf{e} \leftarrow \mathbf{e}^{\prime}\right)$ :

$$
U\left(\mathbf{e} \leftarrow \mathbf{e}^{\prime}\right) \bar{H}^{\prime} U\left(\mathbf{e} \leftarrow \mathbf{e}^{\prime}\right)^{*}=\bar{H} .
$$

Theorem A.2. Assume that $H(\mathbf{p})$ is essentially self-adjoint. Then $H^{\prime}(\mathbf{p})$ is also essentially self-adjoint and $\overline{H(\mathbf{p})}$ is unitarily equivalent to $\overline{H^{\prime}(\mathbf{p})}$ :

$$
U\left(\mathbf{e} \leftarrow \mathbf{e}^{\prime}\right) \overline{H^{\prime}(\mathbf{p})} U\left(\mathbf{e} \leftarrow \mathbf{e}^{\prime}\right)^{*}=\overline{H(\mathbf{p})} .
$$

Remark A.3. The unitary operators $U\left(\mathbf{e} \leftarrow \mathbf{e}^{\prime}\right)$ defined below satisfy the chain rule:

$$
\begin{aligned}
U\left(\mathbf{e} \leftarrow \mathbf{e}^{\prime}\right) & =U\left(\mathbf{e} \leftarrow \mathbf{e}^{\prime \prime}\right) U\left(\mathbf{e}^{\prime \prime} \leftarrow \mathbf{e}^{\prime}\right), \\
U\left(\mathbf{e} \leftarrow \mathbf{e}^{\prime}\right)^{*} & =U\left(\mathbf{e}^{\prime} \leftarrow \mathbf{e}\right) .
\end{aligned}
$$

Proofs of Theorems A.1 and A.2. By the definition of polarization vectors, for each $\mathbf{k} \in \mathbb{R}^{3}$ we have either $\mathbf{e}^{\prime(2)}(\mathbf{k})=\frac{\mathbf{k}}{|\mathbf{k}|} \wedge \mathbf{e}^{\prime(1)}(\mathbf{k})$ or $\mathbf{e}^{\prime(2)}(\mathbf{k})=-\frac{\mathbf{k}}{|\mathbf{k}|} \wedge \mathbf{e}^{\prime(1)}(\mathbf{k})$. Let $O \subset \mathbb{R}^{3}$ be the set of all $\mathbf{k}$ such that $\mathbf{e}^{\prime(2)}(\mathbf{k})=-\frac{\mathbf{k}}{|\mathbf{k}|} \wedge \mathbf{e}^{\prime(1)}(\mathbf{k})$. We define

$$
\mathbf{e}^{\prime \prime(1)}(\mathbf{k}):=\mathbf{e}^{\prime(1)}(\mathbf{k}), \quad \mathbf{e}^{\prime \prime(2)}(\mathbf{k}):= \begin{cases}\mathbf{e}^{(2)}(\mathbf{k}), & \mathbf{k} \in \mathbb{R}^{3} \backslash O \\ -\mathbf{e}^{\prime(2)}(\mathbf{k}), & \mathbf{k} \in O\end{cases}
$$

We define an operator $H^{\prime \prime}$ just as $H$ with $\mathbf{e}^{(\lambda)}$ replaced by $\mathbf{e}^{\prime \prime(\lambda)}, \lambda=1,2$. Let

$$
\mathbf{g}^{\prime}(\mathbf{k}, \lambda ; \mathbf{x}):=\frac{\hat{\rho}(\mathbf{k})}{|\mathbf{k}|^{1 / 2}} \mathbf{e}^{\prime(\lambda)}(\mathbf{k}) e^{-i \mathbf{k} \cdot \mathbf{x}}, \quad \mathbf{g}^{\prime \prime}(\mathbf{k}, \lambda ; \mathbf{x}):=\frac{\hat{\rho}(\mathbf{k})}{|\mathbf{k}|^{1 / 2}} \mathbf{e}^{\prime \prime(\lambda)}(\mathbf{k}) e^{-i \mathbf{k} \cdot \mathbf{x}},
$$

and we set

$$
\mathbf{A}^{\sharp}(\hat{\mathbf{x}}):=\frac{1}{\sqrt{2}} \int_{\mathbb{R}^{3}}^{\oplus} \overline{\left[a\left(\mathbf{g}^{\sharp}(\cdot, \mathbf{x})\right)+a\left(\mathbf{g}^{\sharp}(\cdot, \mathbf{x})\right)^{*}\right]} d \mathbf{x},
$$

where $\sharp$ stands for ' or ". Since $\left(\mathbf{e}^{\prime \prime(1)}(\mathbf{k}), \mathbf{e}^{\prime \prime(2)}(\mathbf{k}), \mathbf{k}\right)$ are right-handed vectors, i.e., $\mathbf{k} \cdot \mathbf{e}^{\prime \prime(1)}(\mathbf{k})=0, \mathbf{e}^{\prime \prime(2)}(\mathbf{k})=\frac{\mathbf{k}}{|\mathbf{k}|} \wedge \mathbf{e}^{\prime \prime(1)}(\mathbf{k})$, there exists $\theta(\mathbf{k}) \in[0,2 \pi)$ such that

$$
\left[\begin{array}{l}
\mathbf{e}^{(1)}(\mathbf{k}) \\
\mathbf{e}^{(2)}(\mathbf{k})
\end{array}\right]=\left[\begin{array}{cc}
\cos \theta(\mathbf{k}) & -\sin \theta(\mathbf{k}) \\
\sin \theta(\mathbf{k}) & \cos \theta(\mathbf{k})
\end{array}\right]\left[\begin{array}{c}
\mathbf{e}^{\prime \prime(1)}(\mathbf{k}) \\
\mathbf{e}^{\prime \prime(2)}(\mathbf{k})
\end{array}\right]
$$

We define a unitary operator $u_{1}$ on $L^{2}\left(\mathbb{R}_{\mathbf{k}}^{3} \times\{1,2\}\right)$ by

$$
\left[\begin{array}{c}
\left(u_{1} f\right)(\mathbf{k}, 1) \\
\left(u_{1} f\right)(\mathbf{k}, 2)
\end{array}\right]:=\left[\begin{array}{cc}
\cos \theta(\mathbf{k}) & -\sin \theta(\mathbf{k}) \\
\sin \theta(\mathbf{k}) & \cos \theta(\mathbf{k})
\end{array}\right]\left[\begin{array}{l}
f(\mathbf{k}, 1) \\
f(\mathbf{k}, 2)
\end{array}\right], \quad \mathbf{k} \in \mathbb{R}^{3} .
$$


The operator $U\left(\mathbf{e} \leftarrow \mathbf{e}^{\prime \prime}\right):=\Gamma\left(u_{1}\right)$ is a unitary operator on $\mathcal{F}_{\text {rad }}$. It is clear that

$$
U\left(\mathbf{e} \leftarrow \mathbf{e}^{\prime \prime}\right) d \Gamma(\omega) U\left(\mathbf{e} \leftarrow \mathbf{e}^{\prime \prime}\right)^{*}=d \Gamma(\omega) .
$$

By the equality $u_{1} \mathbf{g}^{\prime \prime}(\cdot, \mathbf{x})=\mathbf{g}(\cdot, \mathbf{x})$, we have $U\left(\mathbf{e} \leftarrow \mathbf{e}^{\prime \prime}\right) \mathbf{A}^{\prime \prime}(\hat{\mathbf{x}}) U\left(\mathbf{e} \leftarrow \mathbf{e}^{\prime \prime}\right)^{*}=\mathbf{A}(\hat{\mathbf{x}})$. Therefore we get

$$
U\left(\mathbf{e} \leftarrow \mathbf{e}^{\prime \prime}\right) \overline{H^{\prime \prime}} U\left(\mathbf{e} \leftarrow \mathbf{e}^{\prime \prime}\right)^{*}=\overline{U\left(\mathbf{e} \leftarrow \mathbf{e}^{\prime \prime}\right) H^{\prime \prime} U\left(\mathbf{e} \leftarrow \mathbf{e}^{\prime \prime}\right)^{*}}=\bar{H} .
$$

This means that the operator $H^{\prime \prime}$ is essentially self-adjoint and $\overline{H^{\prime \prime}}$ is unitarily equivalent to $\bar{H}$. Next we show that $\overline{H^{\prime \prime}}$ is unitarily equivalent to $\overline{H^{\prime}}$. Let $u_{2}$ be a unitary operator on $L^{2}\left(\mathbb{R}_{\mathbf{k}}^{3} \times\{1,2\}\right)$ such that

$$
\left(u_{2} f\right)(\mathbf{k}, \lambda):= \begin{cases}-f(\mathbf{k}, 2), & \mathbf{k} \in O, \\ f(\mathbf{k}, \lambda), & \text { otherwise. }\end{cases}
$$

It is easy to see that $u_{1} g_{j}^{\prime}(\cdot, \mathbf{x})=g_{j}^{\prime \prime}(\cdot, \mathbf{x}), j=1,2,3$. Then $U\left(\mathbf{e}^{\prime \prime} \leftarrow \mathbf{e}^{\prime}\right):=\Gamma\left(u_{2}\right)$ is a unitary transformation on $\mathcal{F}_{\text {rad }}$, and

$$
U\left(\mathbf{e}^{\prime \prime} \leftarrow \mathbf{e}^{\prime}\right) d \Gamma(\omega) U\left(\mathbf{e}^{\prime \prime} \leftarrow \mathbf{e}^{\prime}\right)^{*}=d \Gamma(\omega)
$$

By the definition of $u_{2}$, the equality $U\left(\mathbf{e}^{\prime \prime} \leftarrow \mathbf{e}^{\prime}\right) \mathbf{A}^{\prime}(\hat{\mathbf{x}}) U\left(\mathbf{e}^{\prime \prime} \leftarrow \mathbf{e}^{\prime}\right)^{*}=\mathbf{A}^{\prime \prime}(\hat{\mathbf{x}})$ holds. Hence

$$
U\left(\mathbf{e}^{\prime \prime} \leftarrow \mathbf{e}^{\prime}\right) \overline{H^{\prime}} U\left(\mathbf{e}^{\prime \prime} \leftarrow \mathbf{e}^{\prime}\right)^{*}=\overline{U\left(\mathbf{e}^{\prime \prime} \leftarrow \mathbf{e}^{\prime}\right) H^{\prime} U\left(\mathbf{e}^{\prime \prime} \leftarrow \mathbf{e}^{\prime}\right)^{*}}=\overline{H^{\prime \prime}}
$$

which implies that $H^{\prime}$ is essentially self-adjoint and $\overline{H^{\prime}}$ is unitarily equivalent to $\overline{H^{\prime \prime}}$. We set

$$
U\left(\mathbf{e} \leftarrow \mathbf{e}^{\prime}\right):=U\left(\mathbf{e} \leftarrow \mathbf{e}^{\prime \prime}\right) U\left(\mathbf{e}^{\prime \prime} \leftarrow \mathbf{e}^{\prime}\right) .
$$

Then $U\left(\mathbf{e} \leftarrow \mathbf{e}^{\prime}\right) \overline{H^{\prime}} U\left(\mathbf{e} \leftarrow \mathbf{e}^{\prime}\right)^{*}=\bar{H}$. Thus Theorem A.1 is proved. The proof of Theorem A.2 is similar.

\section{Appendix B. Remarks on the angular momentum}

As is shown in Appendix A, spectral properties of QED models are independent of the choice of polarization vectors. Hence, in the definition of QED models, usually we do not need to specify them. However, the angular momentum of the electromagnetic field depends on the choice of polarization vectors, since the angular momentum does not commute with $U\left(\mathbf{e} \leftarrow \mathbf{e}^{\prime}\right)$. Therefore, when we discuss an angular momentum, we carefully specify the choice of polarization vectors. One can find the definition of an angular momentum for the electromagnetic field in the textbook [Sp, Section 13.5] (see also [Hi]). In this appendix, we propose an alternate definition. 
Let $(H, \mathbf{e})$ be the pair of a Hamiltonian and polarization vectors.

For each unit vector $\mathbf{j} \in \mathbb{R}^{3}$, we can define specific polarization vectors $\overline{\mathbf{e}}=$ $\left(\overline{\mathbf{e}}^{(1)}, \overline{\mathbf{e}}^{(2)}\right)$ by

$$
\overline{\mathbf{e}}^{(1)}(\mathbf{k}):=\frac{\mathbf{k} \wedge \mathbf{j}}{|\mathbf{k} \wedge \mathbf{j}|}, \quad \overline{\mathbf{e}}^{(2)}(\mathbf{k}):=\frac{\mathbf{k}}{|\mathbf{k}|} \wedge \overline{\mathbf{e}}^{(1)}(\mathbf{k}) .
$$

For the Dirac-Maxwell model $(H, \overline{\mathbf{e}})$, we define the angular momentum around the $\mathbf{j}$-axis by

$$
L_{\mathbf{j}}(\overline{\mathbf{e}}):=d \Gamma(\overrightarrow{\mathbf{j} \cdot \vec{\ell}})
$$

where

$$
\vec{\ell}:=\left(\ell_{1}, \ell_{2}, \ell_{3}\right):=i\left(\nabla_{\mathbf{k}} \wedge \mathbf{k}\right)
$$

is a triplet of self-adjoint operators acting on $L^{2}\left(\mathbb{R}_{\mathbf{k}}^{3} \times\{1,2\}\right)$.

Let $\mathbf{e}=\left(\mathbf{e}^{(1)}, \mathbf{e}^{(2)}\right)$ be any polarization vectors. The angular momentum around the $\mathbf{j}$-axis in the Dirac-Maxwell model $(H, \mathbf{e})$ is defined by

$$
L_{\mathbf{j}}(\mathbf{e}):=U(\mathbf{e} \leftarrow \overline{\mathbf{e}}) L_{\mathbf{j}}(\overline{\mathbf{e}}) U(\mathbf{e} \leftarrow \overline{\mathbf{e}})^{*},
$$

where $U(\overline{\mathbf{e}} \leftarrow \mathbf{e})$ is the unitary operator defined in Appendix A. By the chain rule for $U\left(\mathbf{e} \leftarrow \mathbf{e}^{\prime}\right)$, the angular momentums transform as

$$
L_{\mathbf{j}}(\mathbf{e})=U\left(\mathbf{e} \leftarrow \mathbf{e}^{\prime}\right) L_{\mathbf{j}}\left(\mathbf{e}^{\prime}\right) U\left(\mathbf{e} \leftarrow \mathbf{e}^{\prime}\right)^{*},
$$

where $\mathbf{e}$ and $\mathbf{e}^{\prime}$ are arbitrary polarization vectors.

\section{Appendix C. Some properties of the lowest energy}

In this appendix, we show some properties of $E_{\nu}(\mathbf{p})$ which are used in the proofs of Theorems 4.1-4.4.

Proposition C.1 (Concavity). $E_{\nu}(\mathbf{p})$ is concave in $(\mathbf{p}, m, q) \in \mathbb{R}^{3} \times \mathbb{R} \times \mathbb{R}$.

Proof. See [A2].

Proposition C.2 (Continuity). $E_{\nu}(\mathbf{p}, m)$ is Lipschitz continuous in $(\mathbf{p}, m)$, i.e.,

$$
\left|E_{\nu}(\mathbf{p}, m)-E_{\nu}\left(\mathbf{p}^{\prime}, m^{\prime}\right)\right| \leq \sqrt{\left|\mathbf{p}-\mathbf{p}^{\prime}\right|^{2}+\left|m-m^{\prime}\right|^{2}}, \quad \mathbf{p}, \mathbf{p}^{\prime} \in \mathbb{R}^{3}, m, m^{\prime} \in \mathbb{R} .
$$

Proof. See [A2].

Proposition C.3 (Reflection symmetry in $m$ ). The Hamiltonian $\overline{H_{\nu}(\mathbf{p}, m)}$ is unitarily equivalent to $\overline{H_{\nu}(\mathbf{p},-m)}$. In particular

$$
E_{\nu}(\mathbf{p}, m)=E_{\nu}(\mathbf{p},-m), \quad E_{\nu}(\mathbf{p}, m) \leq E_{\nu}(\mathbf{p}, 0) .
$$


Proof. Let $\gamma_{5}:=-i \alpha_{1} \alpha_{2} \alpha_{3}$. Then $\gamma_{5}$ is a unitary operator and $\gamma_{5} \overline{H_{\nu}(\mathbf{p}, m)} \gamma_{5}^{*}=$ $\overline{H_{\nu}(\mathbf{p},-m)}$. Therefore $E_{\nu}(\mathbf{p}, m)=E_{\nu}(\mathbf{p},-m)$. By Proposition C.1, $m \mapsto E_{\nu}(\mathbf{p}, m)$ is concave. Hence $E_{\nu}(\mathbf{p}, 0)=E_{\nu}\left(\mathbf{p}, \frac{1}{2} m-\frac{1}{2} m\right) \geq E_{\nu}(\mathbf{p}, m)$.

Proposition C.4 (Rotation invariance of the total momentum). Let $T \in O(3)$. Assume that $|\hat{\rho}(\mathbf{k})|=|\hat{\rho}(T \mathbf{k})|$ for a.e. $\mathbf{k} \in \mathbb{R}^{3}$. Then $\overline{H_{\nu}(\mathbf{p})}$ is unitarily equivalent to $\overline{H_{\nu}(T \mathbf{p})}$. In particular, $E_{\nu}(\mathbf{p})=E_{\nu}(T \mathbf{p})$.

Proof. For $T \in O(3)$, we define four $4 \times 4$ matrices by

$$
\beta^{\prime}:=\beta, \quad \alpha_{j}^{\prime}:=\sum_{l=1}^{3} T_{j, l} \alpha_{l}, \quad j=1,2,3 ;
$$

they obey $\left\{\alpha_{j}^{\prime}, \beta^{\prime}\right\}=0,\left\{\alpha_{j}^{\prime}, \alpha_{l}^{\prime}\right\}=2 \delta_{j, l}, j, l=1,2,3$. Then there exists a $4 \times 4$ unitary matrix $u_{T}$ such that (see [T, Lemma 2.25])

$$
u_{T} \alpha_{j} u_{T}^{-1}=\sum_{k=1}^{3} T_{j, k} \alpha_{k}, \quad u_{T} \beta u_{T}^{-1}=\beta .
$$

Therefore $u_{T} \boldsymbol{\alpha} \cdot \mathbf{p} u_{T}^{-1}=\sum_{k, l=1}^{3} T_{l, k} \alpha_{k} p_{l}=\sum_{k, l=1}^{3} \alpha_{k}\left(T^{-1}\right)_{k, l} p_{l}=\boldsymbol{\alpha} \cdot\left(T^{-1} \mathbf{p}\right)$. Similarly, we have

$$
u_{T}(\boldsymbol{\alpha} \cdot d \Gamma(\mathbf{k})) u_{T}^{-1}=\boldsymbol{\alpha} \cdot\left(T^{-1} d \Gamma(\mathbf{k})\right), \quad u_{T} \boldsymbol{\alpha} \cdot \mathbf{A} u_{T}^{-1}=\boldsymbol{\alpha} \cdot\left(T^{-1} \mathbf{A}\right)=(T \boldsymbol{\alpha}) \cdot \mathbf{A} .
$$

We define a rotation operator $\hat{T}$ of photon momentum by

$$
(\hat{T} f)(\mathbf{k}, \lambda)=f\left(T^{-1} \mathbf{k}, \lambda\right), \quad(\mathbf{k}, \lambda) \in \mathbb{R}_{\mathbf{k}}^{3} \times\{1,2\}, f \in L^{2}\left(\mathbb{R}_{\mathbf{k}}^{3} \times\{1,2\}\right) .
$$

Then for all $f \in \operatorname{Dom}\left(k_{j} \hat{T}\right)$,

$$
\hat{T}^{-1} k_{j} \hat{T} f(\mathbf{k}, \lambda)=\left(k_{j} \hat{T} f\right)(T \mathbf{k}, \lambda)=(T \mathbf{k})_{j}(\hat{T} f)(T \mathbf{k}, \lambda)=(T \mathbf{k})_{j} f(\mathbf{k}, \lambda) .
$$

Hence we obtain the operator equality $\hat{T}^{-1} k_{j} \hat{T}=(T \mathbf{k})_{j}, j=1,2,3$. Thus

$$
\begin{aligned}
& \Gamma\left(\hat{T}^{-1}\right) d \Gamma\left(k_{j}\right) \Gamma(\hat{T})=d \Gamma\left((T \mathbf{k})_{j}\right)=(T \cdot d \Gamma(\mathbf{k}))_{j}, \\
& \Gamma\left(\hat{T}^{-1}\right) H_{f}(\nu) \Gamma(\hat{T})=H_{f}(\nu), \\
& \Gamma\left(\hat{T}^{-1}\right) A_{j} \Gamma(\hat{T}),=\Phi_{S}\left(\hat{T}^{-1} g_{j}\right), \quad j=1,2,3,
\end{aligned}
$$

where $\Phi_{S}(\cdot)$ is the Segal field operator (see [RS2, p. 209]) and $g_{j}(\cdot):=g_{j}(\cdot, \mathbf{x}=\mathbf{0}) \in$ $L^{2}\left(\mathbb{R}_{\mathbf{k}}^{3} \times\{1,2\}\right)$. The operator $U:=u_{T} \otimes \Gamma\left(\hat{T}^{-1}\right)$ is a unitary operator on $\mathbb{C}^{4} \otimes \mathcal{F}_{\text {rad }}$ and

$$
\begin{aligned}
U \overline{H_{\nu}(\mathbf{p})} U^{-1} & \overline{\left(\boldsymbol{\alpha} \cdot\left(T^{-1} \mathbf{p}\right)+m \beta+H_{f}(\nu)-\boldsymbol{\alpha} \cdot d \Gamma(\mathbf{k})-q(T \boldsymbol{\alpha}) \cdot \Phi_{S}\left(\hat{T}^{-1} \mathbf{g}\right)\right)} .
\end{aligned}
$$


Note that $T$ is a $3 \times 3$ matrix and $\hat{T}$ is unitary on $L^{2}\left(\mathbb{R}_{\mathbf{k}}^{3} \times\{1,2\}\right)$. Since $T \in O(3)$, we have $(T \boldsymbol{\alpha}) \cdot \Phi_{S}\left(\hat{T}^{-1} \mathbf{g}\right)=\boldsymbol{\alpha} \cdot T^{-1} \Phi_{S}\left(\hat{T}^{-1} \mathbf{g}\right)$, i.e.,

$$
\left(T^{-1} \Phi_{S}\left(\hat{T}^{-1} \mathbf{g}\right)\right)_{j}=\sum_{l=1}^{3}\left(T^{-1}\right)_{j, l} \Phi_{S}\left(\hat{T}^{-1} g_{l}\right), \quad j=1,2,3 .
$$

We define

$$
\mathbf{e}^{\prime(\lambda)}(\mathbf{k})=T^{-1} \mathbf{e}^{(\lambda)}(T \mathbf{k}), \quad(\mathbf{k}, \lambda) \in \mathbb{R}^{3} \times\{1,2\} .
$$

Then $\mathbf{e}^{\prime(1)}$ and $\mathbf{e}^{\prime(2)}$ are polarization vectors: $\mathbf{k} \cdot \mathbf{e}^{\prime(\lambda)}(\mathbf{k})=0, \mathbf{e}^{\prime(\lambda)}(\mathbf{k}) \cdot \mathbf{e}^{\prime(\mu)}(\mathbf{k})=\delta_{\lambda, \mu}$. Since $|\hat{\rho}(\mathbf{k})|=|\hat{\rho}(T \mathbf{k})|$, there exists a Borel measurable function $\mathbf{k} \mapsto \kappa(\mathbf{k}) \in \mathbb{R}$ such that $\hat{\rho}(T \mathbf{k})=e^{i \kappa(\mathbf{k})} \hat{\rho}(\mathbf{k})$ for a.e. $\mathbf{k} \in \mathbb{R}^{3}$. Therefore, we have

$$
\sum_{l=1}^{3}\left(T^{-1}\right)_{j, l} g_{l}(T \mathbf{k}, \lambda)=\frac{e^{i \kappa(\mathbf{k})} \hat{\rho}(\mathbf{k})}{|\mathbf{k}|^{1 / 2}} e_{j}^{\prime(\lambda)}(\mathbf{k}) .
$$

Let $H_{\nu}^{\prime}(\mathbf{p})$ be defined just as $H_{\nu}(\mathbf{p})$ with $\mathbf{e}^{(\lambda)}$ replaced by $\mathbf{e}^{(\lambda)}$. By $($ C.1 $)-($ C.3 $)$, we have

$$
U \overline{H_{\nu}(\mathbf{p})} U^{*}=V \overline{H_{\nu}^{\prime}\left(T^{-1} \mathbf{p}\right)} V^{*},
$$

where $V:=\Gamma\left(e^{i \kappa(\cdot)}\right)$. By Theorem A.2, $\overline{H_{\nu}^{\prime}\left(T^{-1} \mathbf{p}\right)}$ is unitarily equivalent to $\overline{H_{\nu}\left(T^{-1} \mathbf{p}\right)}$. Therefore, $\overline{H(\mathbf{p})}$ is unitarily equivalent to $\overline{H_{\nu}\left(T^{-1} \mathbf{p}\right)}$. Since $\mathbf{p} \in \mathbb{R}^{3}$ is arbitrary, $\overline{H_{\nu}(\mathbf{p})}$ is unitarily equivalent to $\overline{H_{\nu}(T \mathbf{p})}$, and $E_{\nu}(\mathbf{p})=E_{\nu}(T \mathbf{p})$.

If the cutoff function $|\hat{\rho}(\mathbf{k})|$ has reflection symmetry at the origin, the following important inequality holds.

Proposition C.5. Assume that $|\hat{\rho}(\mathbf{k})|=|\hat{\rho}(-\mathbf{k})|$ for almost every $\mathbf{k} \in \mathbb{R}^{3}$. Then

$$
E_{\nu}(\mathbf{p}) \leq E_{\nu}(\mathbf{0}), \quad \mathbf{p} \in \mathbb{R}^{3} \backslash\{\mathbf{0}\} .
$$

Proof. By the assumption $\hat{\rho}(\mathbf{k})=\hat{\rho}(-\mathbf{k})$ for a.e. $\mathbf{k} \in \mathbb{R}^{3}$ and Proposition C.4, we have $E_{\nu}(\mathbf{p})=E_{\nu}(-\mathbf{p}), \mathbf{p} \in \mathbb{R}^{3}$. Using the concavity of $E_{\nu}(\mathbf{p})$ with respect to $\mathbf{p}$, we obtain

$$
E_{\nu}(\mathbf{0})=E_{\nu}\left(\frac{1}{2} \mathbf{p}-\frac{1}{2} \mathbf{p}\right) \geq \frac{1}{2} E_{\nu}(\mathbf{p})+\frac{1}{2} E_{\nu}(-\mathbf{p})=E_{\nu}(\mathbf{p})
$$

for all $\mathbf{p} \in \mathbb{R}^{3}$.

Assuming that $H_{\nu}(\mathbf{0})$ has a ground state, we can obtain the following strict inverse energy inequality:

Proposition C.6. Assume that $|\hat{\rho}(\mathbf{k})|=|\hat{\rho}(-\mathbf{k})|$ for a.e. $\mathbf{k} \in \mathbb{R}^{3}$. If $\overline{H_{\nu}(\mathbf{0})}$ has a ground state, then

$$
E_{\nu}(\mathbf{p})<E_{\nu}(\mathbf{0}) \quad \text { for all } \mathbf{p} \neq \mathbf{0} \text {. }
$$


Remark C.7. When $\nu>0$, the massive Hamiltonian $H_{\nu}(\mathbf{0})$ has a ground state (Lemma 6.1). In the massless case $\nu=0, H(\mathbf{0})$ has a ground state under suitable conditions (see Theorems 4.1, 4.2 and 4.4).

Proof of Proposition C.6. Assume that $E_{\nu}(\mathbf{p})=E_{\nu}(\mathbf{0})$ for some $\mathbf{p} \in \mathbb{R}^{3} \backslash\{\mathbf{0}\}$. Let $\Phi_{\nu}(\mathbf{0})$ be a normalized ground state of $H_{\nu}(\mathbf{0})$. For $t=1,-1$, we have

$$
E_{\nu}(\mathbf{p})=E_{\nu}(t \mathbf{p}) \leq\left\langle\Phi_{\nu}(\mathbf{0}), H_{\nu}(t \mathbf{p}) \Phi_{\nu}(\mathbf{0})\right\rangle=t\left\langle\Phi_{\nu}(\mathbf{0}), \boldsymbol{\alpha} \cdot \mathbf{p} \Phi_{\nu}(\mathbf{0})\right\rangle+E_{\nu}(\mathbf{0}) .
$$

Therefore $\left\langle\Phi_{\nu}(\mathbf{0}), \boldsymbol{\alpha} \cdot \mathbf{p} \Phi_{\nu}(\mathbf{0})\right\rangle=0$, and hence $\left\langle\Phi_{\nu}(\mathbf{0}), H_{\nu}(\mathbf{p}) \Phi_{\nu}(\mathbf{0})\right\rangle=E_{\nu}(\mathbf{0})$ $=E_{\nu}(\mathbf{p})$, which implies $\left\|\left(H_{\nu}(\mathbf{p})-E_{\nu}(\mathbf{p})\right)^{1 / 2} \Phi_{\nu}(\mathbf{0})\right\|=0$, and therefore $\Phi_{\nu}(\mathbf{0})$ is a ground state of $H_{\nu}(\mathbf{p})$. Thus $\boldsymbol{\alpha} \cdot \mathbf{p} \Phi_{\nu}(\mathbf{0})=0$, and we get a contradiction $|\mathbf{p}|^{2} \Phi_{\nu}(\mathbf{0})=0$.

If the cutoff function $\hat{\rho}$ is spherically symmetric, the spectral properties of $\overline{H_{\nu}(\mathbf{p})}$ are independent of the direction of $\mathbf{p}$. The first part of the following proposition immediately follows from Proposition C.4, and the last part from Proposition C.1.

Proposition C.8 (Spherical symmetry in the total momentum). Assume that $|\hat{\rho}(\mathbf{k})|$ is a spherically symmetric function. Then $\overline{H_{\nu}(\mathbf{p})}$ is unitarily equivalent to $\overline{H_{\nu}\left(\mathbf{p}^{\prime}\right)}$ for all $\mathbf{p}^{\prime} \in \mathbb{R}^{3}$ with $|\mathbf{p}|=\left|\mathbf{p}^{\prime}\right|$. In particular $E_{\nu}(\mathbf{p})$ is spherically symmetric with respect to $\mathbf{p}$, and $E_{\nu}(\mathbf{p}) \geq E_{\nu}\left(\mathbf{p}^{\prime}\right)$ if $|\mathbf{p}| \leq\left|\mathbf{p}^{\prime}\right|$.

Proposition C.9 (Massless limit). $E_{\nu}(\mathbf{p})$ is non-decreasing in $\nu \geq 0$ and

$$
\lim _{\nu \rightarrow+0} E_{\nu}(\mathbf{p})=E_{0}(\mathbf{p})
$$

Proof. Let $\nu \geq \nu^{\prime} \geq 0$. Then $H_{\nu}(\mathbf{p}) \geq H_{\nu^{\prime}}(\mathbf{p})$ in the sense of quadratic forms on $\mathcal{D}:=\operatorname{Dom}\left(H_{f}\right) \cap \operatorname{Dom}\left(N_{f}\right)$. Therefore $\nu \mapsto E_{\nu}(\mathbf{p})$ is non-decreasing: $E_{\nu}(\mathbf{p}) \geq$ $E_{\nu^{\prime}}(\mathbf{p})$. It is easy to see that for all $\Psi \in \mathcal{D}, H_{\nu}(\mathbf{p}) \Psi \rightarrow H(\mathbf{p}) \Psi$ as $\nu \rightarrow 0$. Since $\mathcal{D}$ is a common core for all $H_{\nu}(\mathbf{p})$, we have $H_{\nu}(\mathbf{p}) \rightarrow H(\mathbf{p})$ in the strong resolvent sense (see [RS1, Theorem VIII.25]). Using a property of strongly convergent operators [RS1, Theorem VIII.24], we conclude that $E_{\nu}(\mathbf{p}) \rightarrow E(\mathbf{p})$ as $\nu \rightarrow+0$.

By Proposition C.2,

$$
0 \leq E_{\nu}(\mathbf{p}-\mathbf{k})-E_{\nu}(\mathbf{p})+|\mathbf{k}|, \quad \mathbf{p}, \mathbf{k} \in \mathbb{R}^{3} .
$$

The function $\mathbf{k} \mapsto E_{\nu}(\mathbf{p}-\mathbf{k})-E_{\nu}(\mathbf{p})+|\mathbf{k}|$ plays the role of a dispersion relation in the low-energy Dirac polaron. 
Theorem C.10. Let $\nu \geq 0$. Assume that $\hat{\rho}$ is spherically symmetric. Suppose that $E_{\nu}(\mathbf{p}, m)<E_{\nu}(\mathbf{p}, 0)$. Then, for $\mathbf{p} \neq \mathbf{0}$,

$$
E_{\nu}(\mathbf{p}-\mathbf{k}, m)-E_{\nu}(\mathbf{p}, m)+|\mathbf{k}| \geq \begin{cases}|\mathbf{k}| & \text { if }|\mathbf{p}-\mathbf{k}| \leq|\mathbf{p}|, \\ \left(1-b_{\nu}(\mathbf{p})\right)|\mathbf{k}| & \text { if }|\mathbf{p}| \leq|\mathbf{p}-\mathbf{k}| \leq 2|\mathbf{p}|, \\ \left(1-b_{\nu}(\mathbf{p})\right)|\mathbf{p}| & \text { if } 2|\mathbf{p}| \leq|\mathbf{p}-\mathbf{k}|,\end{cases}
$$

where

$$
b_{\nu}(\mathbf{p}):=\frac{E_{\nu}(\mathbf{p}, m)-E_{\nu}(2 \mathbf{p}, m)}{|\mathbf{p}|}<1
$$

In the case $\mathbf{p}=\mathbf{0}$, for all constant $P>0$,

$$
E_{\nu}(\mathbf{k}, m)-E_{\nu}(\mathbf{0}, m)+|\mathbf{k}| \geq \begin{cases}\frac{a_{\nu}(P)}{P}|\mathbf{k}| & \text { if }|\mathbf{k}| \leq P \\ a_{\nu}(P) & \text { if }|\mathbf{k}|>P\end{cases}
$$

where

$$
a_{\nu}(P):=\left.\left(E_{\nu}(\mathbf{k}, m)-E_{\nu}(\mathbf{0}, m)+|\mathbf{k}|\right)\right|_{|\mathbf{k}|=P}
$$

is a strictly positive constant.

Remark C.11. The idea of the proof of Theorem C.10 was developed in [LMS].

Proof of Theorem C.10. Before proving Theorem C.10, we prove the following lemma:

Lemma C.12. Let $\nu \geq 0$. Assume that $E_{\nu}(\mathbf{p}, m)<E_{\nu}(\mathbf{p}, 0)$. Then

$$
E_{\nu}(\mathbf{p}-\mathbf{k}, m)-E_{\nu}(\mathbf{p}, m)+|\mathbf{k}|>0, \quad \mathbf{k} \in \mathbb{R}^{3} \backslash\{\mathbf{0}\} .
$$

Proof. First we prove (C.5) for positive $\nu>0$. We fix $m \neq 0$ and $\mathbf{p} \in \mathbb{R}^{3}$. Suppose that

$$
E_{\nu}(\mathbf{p}-\mathbf{k})-E_{\nu}(\mathbf{p})+|\mathbf{k}|=0
$$

for some $\mathbf{k} \in \mathbb{R}^{3} \backslash\{\mathbf{0}\}$. Let $\Phi_{\nu}(\mathbf{p}-\mathbf{k})$ be a normalized ground state of $H_{\nu}(\mathbf{p}-\mathbf{k})$ (see Lemma 6.1). Then

$$
\begin{aligned}
E_{\nu}(\mathbf{p}-\mathbf{k}) & =\left\langle\Phi_{\nu}(\mathbf{p}-\mathbf{k}), \overline{H_{\nu}(\mathbf{p}-\mathbf{k})} \Phi_{\nu}(\mathbf{p}-\mathbf{k})\right\rangle \\
& =\left\langle\Phi_{\nu}(\mathbf{p}-\mathbf{k}), \overline{H_{\nu}(\mathbf{p})} \Phi_{\nu}(\mathbf{p}-\mathbf{k})\right\rangle-\left\langle\Phi_{\nu}(\mathbf{p}-\mathbf{k}), \boldsymbol{\alpha} \cdot \mathbf{k} \Phi_{\nu}(\mathbf{p}-\mathbf{k})\right\rangle \\
& \geq E_{\nu}(\mathbf{p})-|\mathbf{k}|
\end{aligned}
$$

Hence, by assumption (C.6) we have $\left\langle\Phi_{\nu}(\mathbf{p}-\mathbf{k}), \overline{H_{\nu}(\mathbf{p})} \Phi_{\nu}(\mathbf{p}-\mathbf{k})\right\rangle=E_{\nu}(\mathbf{p})$ and $\left\langle\Phi_{\nu}(\mathbf{p}-\mathbf{k}), \boldsymbol{\alpha} \cdot \mathbf{k} \Phi_{\nu}(\mathbf{p}-\mathbf{k})\right\rangle=|\mathbf{k}|$, which implies that $\Phi_{\nu}(\mathbf{p}-\mathbf{k})$ is a ground state 
of both $\overline{H_{\nu}(\mathbf{p})}$ and $-\boldsymbol{\alpha} \cdot \mathbf{k}$. Since $\mathbf{k} \neq \mathbf{0}$, we have $\left\langle\Phi_{\nu}(\mathbf{p}-\mathbf{k}), \beta \Phi_{\nu}(\mathbf{p}-\mathbf{k})\right\rangle=0$, because $\boldsymbol{\alpha} \cdot \mathbf{k} \beta=-\beta \boldsymbol{\alpha} \cdot \mathbf{k}$. In what follows, to emphasize $m$-dependence, we write $H_{\nu}(\mathbf{p}-\mathbf{k}, m)$ and $\Phi_{\nu}(\mathbf{p}-\mathbf{k}, m)$ for $H_{\nu}(\mathbf{p}-\mathbf{k})$ and $\Phi_{\nu}(\mathbf{p}-\mathbf{k})$, respectively. By using the above facts, we have

$$
E_{\nu}(\mathbf{p}, m)=\left\langle\Phi_{\nu}(\mathbf{p}-\mathbf{k}, m), \overline{H_{\nu}(\mathbf{p}, 0)} \Phi_{\nu}(\mathbf{p}-\mathbf{k}, m)\right\rangle \geq E_{\nu}(\mathbf{p}, 0),
$$

which contradicts the inequality $E_{\nu}(\mathbf{p}, m)<E_{\nu}(\mathbf{p}, 0)$.

Next, we handle the case $\nu=0$. Suppose that there exists a vector $\mathbf{k} \in \mathbb{R}^{3} \backslash\{\mathbf{0}\}$ such that $E(\mathbf{p}-\mathbf{k}, m)-E(\mathbf{p}, m)+|\mathbf{k}|=0$. It is not difficult to see that

$$
\lim _{\nu \rightarrow+0}\left\langle\Phi_{\nu}(\mathbf{p}-\mathbf{k}, m), \overline{H(\mathbf{p}-\mathbf{k}, m)} \Phi_{\nu}(\mathbf{p}-\mathbf{k}, m)\right\rangle=E(\mathbf{p}-\mathbf{k}, m) .
$$

By these equations, we have

$$
\begin{aligned}
& \lim _{\nu \rightarrow+0}\left\langle\Phi_{\nu}(\mathbf{p}-\mathbf{k}, m), \boldsymbol{\alpha} \cdot \mathbf{k} \Phi_{\nu}(\mathbf{p}-\mathbf{k}, m)\right\rangle=|\mathbf{k}| \\
& \lim _{\nu \rightarrow+0}\left\langle\Phi_{\nu}(\mathbf{p}-\mathbf{k}, m), \overline{H(\mathbf{p}, m)} \Phi_{\nu}(\mathbf{p}-\mathbf{k}, m)\right\rangle=E(\mathbf{p}, m) .
\end{aligned}
$$

Equation (C.7) implies that

$$
\lim _{\nu \rightarrow+0}(|\mathbf{k}|-\boldsymbol{\alpha} \cdot \mathbf{k}) \Phi_{\nu}(\mathbf{p}-\mathbf{k}, m)=0 .
$$

Therefore $\lim _{\nu \rightarrow+0}\left\langle\Phi_{\nu}(\mathbf{p}-\mathbf{k}, m), \beta \Phi_{\nu}(\mathbf{p}-\mathbf{k}, m)\right\rangle=0$. This fact and (C.8) imply $E(\mathbf{p}, m)=E(\mathbf{p}, 0)$, which contradicts $E(\mathbf{p}, m)<E(\mathbf{p}, 0)$.

We fix a vector $\mathbf{p}$ such that $E_{\nu}(\mathbf{p}, m)<E_{\nu}(\mathbf{p}, 0)$. Since $\hat{\rho}$ is spherically symmetric, by Proposition C.8 the function

$$
G_{\nu}(|\mathbf{k}|):=E_{\nu}(\mathbf{0})-E_{\nu}(\mathbf{k}), \quad \mathbf{k} \in \mathbb{R}^{3}
$$

is non-decreasing, convex with respect to $|\mathbf{k}|$, and

$$
0 \leq G_{\nu}(|\mathbf{k}|) \leq|\mathbf{k}|, \quad \mathbf{k} \in \mathbb{R}^{3}
$$

Since $G_{\nu}(s)$ is convex, $G_{\nu}(s)$ has a right derivative $G_{\nu}^{+{ }^{\prime}}(s)$ :

$$
G_{\nu}^{+^{\prime}}(s):=\lim _{h \rightarrow+0}\left[G_{\nu}(s+h)-G_{\nu}(s)\right] / h .
$$

First we show that

$$
G_{\nu}^{+{ }^{\prime}}(s)<1, \quad 0 \leq s \leq|\mathbf{p}|
$$


Since $G_{\nu}(s)$ is convex and $0 \leq G_{\nu}(s) \leq s, G_{\nu}^{+^{\prime}}(s)$ is a non-decreasing function of $s$. If $G_{\nu}^{+{ }^{\prime}}\left(s_{0}\right)>1$ for a constant $s_{0} \geq 0$, then $G_{\nu}^{+^{\prime}}(s)>1$ for all $s \geq s_{0}$, and

$$
G_{\nu}(s)=\int_{s_{0}}^{s} G_{\nu}^{+^{\prime}}(t) d t+\int_{0}^{s_{0}} G_{\nu}^{+^{\prime}}(t) d t \geq\left(s-s_{0}\right) G_{\nu}^{+^{\prime}}\left(s_{0}\right)+\int_{0}^{s_{0}} G_{\nu}^{+^{\prime}}(t) d t
$$

for all $s>s_{0}$. This contradicts (C.9). Thus, $G_{\nu}^{+^{\prime}}(s) \leq 1$ for all $s \geq 0$. Let $s_{1} \geq 0$ be a point such that $G_{\nu}^{+{ }^{\prime}}\left(s_{1}\right)=1$ and $G_{\nu}^{+^{\prime}}\left(s_{1}-\epsilon\right)<1$ for all $0<\epsilon \leq s_{1}$. If $|\mathbf{p}|<s_{1}$, (C.10) is trivial, so we consider the case $|\mathbf{p}| \geq s_{1}$. Note that $G_{\nu}^{+^{\prime}}(s)=1$ for all $s \geq s_{1}$. Hence $G_{\nu}(s)$ is a linear function of $s$ if $s \geq s_{1}$ :

$$
G_{\nu}(s)=s+C, \quad s \geq s_{1},
$$

where $C$ is a negative constant. By this equality, we have

$$
E_{\nu}(\mathbf{p}-\mathbf{k})-E_{\nu}(\mathbf{p})+|\mathbf{k}|=-|\mathbf{p}-\mathbf{k}|+|\mathbf{p}|+|\mathbf{k}|,
$$

for all $\mathbf{p}$ and $\mathbf{k}$ such that $|\mathbf{p}-\mathbf{k}| \geq s_{1}$ and $|\mathbf{p}| \geq s_{1}$. We choose $\mathbf{k}=-C \mathbf{p}$ for a constant $C>s_{1} /|\mathbf{p}|$. Then

$$
E_{\nu}(\mathbf{p}-\mathbf{k})-E_{\nu}(\mathbf{p})+|\mathbf{k}|=0
$$

contrary to Lemma C.12. Therefore $G_{\nu}^{{ }^{\prime}}(s)<1$ for all $0 \leq s \leq|\mathbf{p}|$.

Next, by using this inequality, we prove Theorem C.10. By (C.10) and convexity of $G_{\nu}$,

$$
c_{\nu}(\mathbf{p}):=\frac{G_{\nu}(|\mathbf{p}|)}{|\mathbf{p}|} \leq b_{\nu}(\mathbf{p})<1 .
$$

We define

$$
\begin{aligned}
\mathcal{C}:=\left\{J: \mathbb{R}_{+} \rightarrow \mathbb{R}_{+} \mid J \text { is convex, } 0 \leq J(s) \leq s(s \geq 0)\right. & \\
& \left.J(|\mathbf{p}|)=G_{\nu}(|\mathbf{p}|), J(2|\mathbf{p}|)=G_{\nu}(2|\mathbf{p}|)\right\} .
\end{aligned}
$$

Then

$$
\begin{aligned}
E_{\nu}(\mathbf{p}-\mathbf{k})-E_{\nu}(\mathbf{p})+|\mathbf{k}| & =|\mathbf{k}|+G_{\nu}(\mathbf{p})-G_{\nu}(\mathbf{p}-\mathbf{k}) \\
& \geq|\mathbf{k}|+G_{\nu}(\mathbf{p})-\sup _{J \in \mathcal{C}} J(\mathbf{p}-\mathbf{k})=: I .
\end{aligned}
$$

The maximal function in $\mathcal{C}$ is given by the following linear interpolation:

$$
J_{\max }(s):= \begin{cases}c_{\nu}(\mathbf{p}) s & \text { if } s \leq|\mathbf{p}| \\ b_{\nu}(\mathbf{p})(s-|\mathbf{p}|)+G_{\nu}(|\mathbf{p}|) & \text { if }|\mathbf{p}| \leq s \leq 2|\mathbf{p}|, \\ s-2|\mathbf{p}|+G_{\nu}(2|\mathbf{p}|) & \text { if } 2|\mathbf{p}| \leq|\mathbf{p}-\mathbf{k}|\end{cases}
$$


Hence

$$
\begin{aligned}
& I \geq|\mathbf{k}|+G_{\nu}(|\mathbf{p}|)- \begin{cases}c_{\nu}(\mathbf{p})|\mathbf{p}-\mathbf{k}| & \text { if }|\mathbf{p}-\mathbf{k}| \leq|\mathbf{p}|, \\
b_{\nu}(\mathbf{p})(|\mathbf{p}-\mathbf{k}|-|\mathbf{p}|)+G_{\nu}(|\mathbf{p}|) & \text { if }|\mathbf{p}| \leq|\mathbf{p}-\mathbf{k}| \leq 2|\mathbf{p}|, \\
|\mathbf{p}-\mathbf{k}|-2|\mathbf{p}|+G_{\nu}(2|\mathbf{p}|) & \text { if } 2|\mathbf{p}| \leq|\mathbf{p}-\mathbf{k}| .\end{cases} \\
&= \begin{cases}|\mathbf{k}|+c_{\nu}(\mathbf{p})(|\mathbf{p}|-|\mathbf{p}-\mathbf{k}|) & \text { if }|\mathbf{p}-\mathbf{k}| \leq|\mathbf{p}|, \\
|\mathbf{k}|-b_{\nu}(\mathbf{p})(|\mathbf{p}-\mathbf{k}|-|\mathbf{p}|) & \text { if }|\mathbf{p}| \leq|\mathbf{p}-\mathbf{k}| \leq 2|\mathbf{p}|, \\
|\mathbf{k}|-|\mathbf{p}-\mathbf{k}|+\left(2-b_{\nu}(\mathbf{p})\right)|\mathbf{p}| & \text { if } 2|\mathbf{p}| \leq|\mathbf{p}-\mathbf{k}| .\end{cases}
\end{aligned}
$$

Using the triangle inequality, one can obtain the desired estimate.

Finally, we prove (C.4). Since $G_{\nu}^{+^{\prime}}(0)<1$ and $G_{\nu}$ is convex, the constant $a_{\nu}(P)$ is strictly positive for all $P>0$. It is easy to see that

$$
{G_{\nu}^{+}}^{\prime}(s) \leq \frac{G_{\nu}(P)}{P}=\frac{-a_{\nu}(P)+P}{P}, \quad s \leq P .
$$

Hence

$$
\begin{aligned}
E_{\nu}(\mathbf{k})-E_{\nu}(\mathbf{0})+|\mathbf{k}| & =|\mathbf{k}|-G_{\nu}(|\mathbf{k}|)=\int_{0}^{|\mathbf{k}|}\left(1-G_{\nu}^{+^{\prime}}(s)\right) d s \\
& \geq \begin{cases}\int_{0}^{|\mathbf{k}|}\left(1-\frac{G_{\nu}(P)}{P}\right) d s & \text { if }|\mathbf{k}| \leq P, \\
\int_{0}^{P}\left(1-\frac{G_{\nu}(P)}{P}\right) d s+\int_{P}^{|\mathbf{k}|}\left(1-G_{\nu}^{+^{\prime}}(s)\right) d s & \text { if }|\mathbf{k}|>P,\end{cases} \\
& \geq \begin{cases}\left(a_{\nu}(P) / P\right)|\mathbf{k}| & \text { if }|\mathbf{k}| \leq P, \\
a_{\nu}(P) & \text { if }|\mathbf{k}|>P .\end{cases}
\end{aligned}
$$

This completes the proof.

\section{Acknowledgements}

This work was partly supported by KAKENHI Y22740087, and was performed through the Program for Dissemination of Tenure-Track System funded by the Ministry of Education and Science, Japan. I would like to thank A. Arai for his advice, discussions and encouragement. I am grateful to T. Miyao and F. Hiroshima for their advice. I also thank the unknown referees for a lot of useful comments.

\section{References}

[A1] A. Arai, Fundamental properties of the Hamiltonian of a Dirac particle coupled to the quantized radiation field, Hokkaido Univ. Preprint Series in Math. (1999), no. 447.

[A2] , A particle-field Hamiltonian in relativistic quantum electrodynamics, J. Math. Phys. 41 (2000), 4271-4283. Zbl 0973.81137 MR 1765584 
[A3] Non-relativistic limit of a Dirac-Maxwell operator in relativistic quantum electrodynamics, Rev. Math. Phys. 15 (2003), 245-270. Zbl 1081.81114 MR 1981623

[A4] Non-relativistic limit of a Dirac polaron in relativistic quantum electrodynamics, Lett. Math. Phys. 77 (2006), 283-290. Zbl 1160.81484 MR 2260374

[B] J. D. Bjorken and S. D. Drell, Relativistic quantum mechanics, McGraw-Hill, New York, 1965. Zbl 0184.54201 MR 0187642

[C] T. Chen, Operator-theoretic infrared renormalization and construction of dressed 1particle states in non-relativistic QED, arXiv:math-ph/0108021 (2001).

[Ge] C. Gérard, On the existence of ground states for massless Pauli-Fierz Hamiltonians, Ann. Henri Poincaré 1 (2000), 443-459. Zbl 1004.81012 MR 1777307

[GLL] M. Griesemer, E. H. Lieb, and M. Loss, Ground states in non-relativistic quantum electrodynamics, Invent. Math. 145 (2001), 557-595. Zbl 1044.81133 MR 1856401

[He] W. Heitler, The quantum theory of radiation, Oxford Univ. Press, 1954. Zbl 1254.01048

[Hi] F. Hiroshima, Fiber Hamiltonians in non-relativistic quantum electrodynamics, J. Funct. Anal. 252 (2007), 314-355. Zbl 1129.81030 MR 2357359

[LL] E. H. Lieb and M. Loss, Analysis, Grad. Stud. in Math. 14, Amer. Math. Soc., 2001. Zbl 0966.26002 MR 1817225

[LMS] M. Loss, T. Miyao, and H. Spohn, Lowest energy states in nonrelativistic QED: atoms and ions in motion, J. Funct. Anal. 243 (2007), 353-393. Zbl 1118.81080 MR 2289693

[RS1] M. Reed and B. Simon, Methods of modern mathematical physics. I. Functional analysis, Academic Press, New York, 1972. Zbl 0242.46001 MR 0493419

[RS2] Methods of modern mathematical physics. II. Fourier analysis, self-adjointness, Academic Press, New York, 1975. Zbl 0308.47002 MR 0493420

[S1] I. Sasaki, Ground state energy of the polaron in the relativistic quantum electrodynamnics, J. Math. Phys. 46 (2005), 102307, 6 pp. Zbl 1111.81343 MR 2178579

[S2] Ground state of the polaron in the relativistic quantum electrodynamics, RIMS Kokyuroku 1510 (2006), 87-103.

[Sp] H. Spohn, Dynamics of charged particles and their radiation field, Cambridge Univ. Press, Cambridge, 2004. Zbl 1078.81004 MR 2097788

[SZ] E. Stockmeyer and H. Zenk, Dirac operators coupled to the quantized radiation field: Essential self-adjointness à la Chernoff, Lett. Math. Phys. 83 (2008), 59-68. Zbl 05248429 MR 2377946

[T] B. Thaller, The Dirac equation, Texts Monogr. Phys., Springer, Berlin, 1992. Zbl 0765.47023 MR 1219537 